Supporting Information

\title{
Application of One-Pot Three-Component Condensation Reaction for the Synthesis of New Organo Phosphorus-Sulfur Macrocycles
}

\author{
Guoxiong Hua, David B. Cordes, Alexandra M. Z. Slawin and J. Derek Woollins*
}

\section{Experimental Section}

Unless otherwise stated, all reactions were carried out under on oxygen free nitrogen atmosphere using predried solvents and standard Schlenk techniques, subsequent chromatographic and work up procedures were performed in air. All chemicals and Lawesson's reagent were purchased from Sigma-Aldrich without further purification. 2,4-diferrocenyl-1,3,2,4-diathiadiphosphetane 2,4-disulfide $[\mathrm{FcP}(\mu-\mathrm{S}) \mathrm{S}]_{2}$ (FcLR), was prepared by using the literature method. ${ }^{\mathrm{s} 1, \mathrm{~s} 2}{ }^{1} \mathrm{H}(400.1 \mathrm{MHz}),{ }^{13} \mathrm{C}(100.6 \mathrm{MHz})$ and ${ }^{31} \mathrm{P}-\left\{{ }^{1} \mathrm{H}\right\}(162.0 \mathrm{MHz}) \mathrm{NMR}$ spectra were recorded at $25{ }^{\circ} \mathrm{C}$ (unless stated otherwise) on Bruker Advance II 400. IR spectra were recorded as $\mathrm{KBr}$ pellets in the range of $4000-250 \mathrm{~cm}^{-1}$ on a Perkin-Elmer 2000 FTIR/Raman spectrometer. Mass spectrometry was performed by the EPSRC National Mass Spectrometry Service Centre, Swansea. X-ray diffraction data for compound 14 was collected at $125 \mathrm{~K}$ using the St Andrews Automated Robotic Diffractometer (STANDARD), ${ }^{\mathrm{s} 3}$ consisting of a sealed-tube generator (Rigaku, Houston, USA) equipped with a SHINE monochromator [Mo K $\alpha$ radiation $(\lambda=0.71075 \AA)$ ], and a Saturn 724 CCD area detector, coupled with a Microglide goniometer head and an ACTOR SM robotic sample changer. Diffraction data for compounds 10a, 10b, 11, 12a and 12b were collected at either $93 \mathrm{~K}$ or $173 \mathrm{~K}$ (all other crystals) by using a Rigaku FR-X Ultrahigh Brilliance Microfocus RA generator/confocal optics and Rigaku XtaLAB P200 system, with Mo K $\alpha$ radiation $(\lambda=0.71075 \AA)$. Intensity data were collected using $\omega$ steps accumulating area detector images spanning at least a hemisphere of reciprocal space. All data were corrected for Lorentz polarization effects. A multi-scan absorption correction was applied by using CrystalClear. ${ }^{\text {s4,s5 }}$ Structures were solved by either charge-flipping (SUPERFLIP) ${ }^{\mathrm{s} 6}$ or direct (SIR2004, SIR2011) ${ }^{\mathrm{s} 7,88}$ methods, and refined by full-matrix leastsquares against $\mathrm{F}^{2}$ (SHELXL-2013). ${ }^{\mathrm{s} 9}$ Non-hydrogen atoms were refined anisotropically, and hydrogen atoms were refined using a riding model. All calculations were performed using the CrystalStructure interface. ${ }^{\mathrm{s} 10}$

\section{Synthesis and Characterization of Heterocycles 1-12 and 14}


General Procedure for Preparation of Macrocycles 1-12 and 14. A suspension of alkenyl- or aryl-dithiol (1.0 mmol), triethylamine $(2.0 \mathrm{mmol}), \mathbf{L R}(0.44 \mathrm{~g}, 1.0 \mathrm{mmol})$ or FcLR $(0.56 \mathrm{~g}, 1.0 \mathrm{mmol})$ and dihaloalkane $(1.0 \mathrm{mmol})$ in dry THF $(60 \mathrm{~mL})$ was stirred under $\mathrm{N}_{2}$ gas atmosphere at ambient temperature for $24 \mathrm{~h}$ leading to a reddish yellow or pale-yellow suspension. Upon filtering to remove insoluble solid the filtrate was dried under reduced pressure. The residue was dissolved in dichloromethane ( $c a .2 \mathrm{~mL})$ and was loaded onto a silica gel column (dichloromethane as eluent) to give the macrocycles. Another unexpected nine-membered ring 4 was also obtained in respective $25 \%$ and $30 \%$ yields in the cases of 5 and 9. Two diastereomers were separated completely in the cases of $\mathbf{1 0}$ and $\mathbf{1 2}$.

Compound 1. White solid ( $80 \%$ yield). Two diastereoisomers were found in ca. 1:1 intensity ratio. Selected IR (KBr, cm $\left.{ }^{-1}\right)$ : 1590(s), 1564(m), 1495(s), 1407(m), 1296(m), 1260(s), 1179(m), 1098(s), 1025(m), 829(m), 701(s), 618(m), 528(s). ${ }^{1} \mathrm{H}$ NMR $\left(\mathrm{CD}_{2} \mathrm{Cl}_{2}, \delta\right)$, 8.03-7.71 (m, 8H), 8.03-7.71 (m, 8H), 7.29-6.88 (m, 8H), 4.09$3.96(\mathrm{~m}, 8 \mathrm{H}), 3.78(\mathrm{~s}, 6 \mathrm{H}), 3.76(\mathrm{~s}, 6 \mathrm{H}) \mathrm{ppm} .{ }^{13} \mathrm{C} \mathrm{NMR}\left(\mathrm{CD}_{2} \mathrm{Cl}_{2}, \delta\right), 165.4,164.2,134.9,133.7,133.6,133.4$, 131.8, 131.7, 129.1, 128.8, 115.0, 114.9, 114.8, 114.7, 56.1, 56.0, 35.9, 35.7 ppm. ${ }^{31} \mathrm{P}$ NMR $\left(\mathrm{CD}_{2} \mathrm{Cl}_{2}, \delta\right), 95.1$ and $95.0 \mathrm{ppm}$. Mass spectrum $\left(\mathrm{EI}^{+}, \mathrm{m} / \mathrm{z}\right), 656[\mathrm{M}]^{+}$. Accurate mass measurement $\left[\mathrm{EI}^{+}, \mathrm{m} / \mathrm{z}\right]: 655.9196[\mathrm{M}]^{+}$, calculated mass for $\mathrm{C}_{24} \mathrm{H}_{22} \mathrm{~N}_{2} \mathrm{O}_{2} \mathrm{P}_{2} \mathrm{~S}_{7}$ : 655.9201 .

Compound 2. White solid (60\% yield). Two diastereomers were found in ca. 1:1 intensity ratio. Selected IR (KBr, cm $\left.{ }^{-1}\right)$ : 1590(vs), 1564(m), 1495(s), 440(m), 1407(m), 1295(m), 1259(vs), 1179(s), 1097(vs), 1024(s), 828(m), 800(m), 699(s), 618(m), 527(s), 444(m). ${ }^{1} \mathrm{H}$ NMR $\left(\mathrm{CD}_{2} \mathrm{Cl}_{2}, \delta\right), 7.97-7.84(\mathrm{~m}, 2 \mathrm{H}), 7.38-6.98$ (m, $22 \mathrm{H}), 4.51-3.86(\mathrm{~m}, 8 \mathrm{H}), 3.94(\mathrm{~s}, 6 \mathrm{H}), 3.93(\mathrm{~s}, 6 \mathrm{H}) \mathrm{ppm} .{ }^{13} \mathrm{C} \mathrm{NMR}\left(\mathrm{CD}_{2} \mathrm{Cl}_{2}, \delta\right), 164.4,164.2,138.4,138.3$, $133.9,133.7,133.5,133.3,131.9,131.8,131.2$, 130.8, 130.0, 129.9, 129.4, 129.1, 129.0, 128.8, 56.1, 55.9, 38.9, 38.1 ppm. ${ }^{31} \mathrm{P}$ NMR $\left(\mathrm{CD}_{2} \mathrm{Cl}_{2}, \delta\right), 83.7$ and $82.7 \mathrm{ppm}$. Mass spectrum $\left(\mathrm{EI}^{+}, \mathrm{m} / \mathrm{z}\right), 656[\mathrm{M}]^{+}$. Accurate mass measurement $\left[\mathrm{EI}^{+}, \mathrm{m} / \mathrm{z}\right]: 655.9198[\mathrm{M}]^{+}$, calculated mass for $\mathrm{C}_{24} \mathrm{H}_{22} \mathrm{~N}_{2} \mathrm{O}_{2} \mathrm{P}_{2} \mathrm{~S}_{7}: 655.9201$.

Compound 3. White solid (58\% yield). Two diastereomers were found in $c a$. 1:2 intensity ratio. Selected IR $\left(\mathrm{KBr}, \mathrm{cm}^{-1}\right):$ 1591(s), 1564(m), 1495(s), 1459(m), 1406(m), 1293(m), 1256(s), 1178(s), 1098(s), 1023(s), 828(m), 799(m), 687(s), 618(m), 533(s), 501(m). ${ }^{1} \mathrm{H}$ NMR $\left(\mathrm{CD}_{2} \mathrm{Cl}_{2}, \delta\right), 7.89-7.86(\mathrm{~m}, 8 \mathrm{H}), 7.47-6.93$ (m, $16 \mathrm{H}), 4.44-4.33(\mathrm{~m}, 4 \mathrm{H}), 4.22-4.02(\mathrm{~m}, 4 \mathrm{H}), 3.84(\mathrm{~s}, 6 \mathrm{H}), 3.82(\mathrm{~s}, 6 \mathrm{H}), 3.72-3.65(\mathrm{~m}, 4 \mathrm{H}), 3.18-2.29(\mathrm{~m}, 4 \mathrm{H})$ ppm. ${ }^{13} \mathrm{C}$ NMR $\left(\mathrm{CD}_{2} \mathrm{Cl}_{2}, \delta\right), 163.2,137.2,135.9,133.0,132.8,131.9,131.7,130.7,130.2,129.9,129.5,129.3$, 128.3, 114.3, 114.0, 55.7, 42.8, 37.7, 34.1, 25.3 ppm. ${ }^{31} \mathrm{P} \mathrm{NMR}\left(\mathrm{CD}_{2} \mathrm{Cl}_{2}, \delta\right), 79.9$ and 79.7 ppm. Mass spectrum $\left(\mathrm{EI}^{+}, \mathrm{m} / \mathrm{z}\right), 600[\mathrm{M}]^{+}$. Accurate mass measurement $\left[\mathrm{EI}^{+}, \mathrm{m} / \mathrm{z}\right]: 599.9729[\mathrm{M}]^{+}$, calculated mass for $\mathrm{C}_{24} \mathrm{H}_{26} \mathrm{O}_{2} \mathrm{P}_{2} \mathrm{~S}_{6}: 599.9727$. 
Compound 5. Yellow solid (53\% yield). Two diastereomers were found in ca. 1:1 intensity ratio. Selected IR $\left(\mathrm{KBr}, \mathrm{cm}^{-1}\right):$ 1484(m), 1408(m), 1258(m), 1168(s), 1105(m), 1022(s), 1000(m), 822(s), 766(m), 734(m), 672(vs), 616(m), 531(m), 474(vs). ${ }^{1} \mathrm{H}$ NMR $\left(\mathrm{CD}_{2} \mathrm{Cl}_{2}, \delta\right), 7.42-7.29(\mathrm{~m}, 8 \mathrm{H})$, 4.77-4.74 (m, 8H), 4.67-4.60 (m, $8 \mathrm{H}), 4.43(\mathrm{~s}, 10 \mathrm{H}), 4.40(\mathrm{~s}, 10 \mathrm{H}), 4.33-4.27(\mathrm{~m}, 4 \mathrm{H}), 4.11-4.03(\mathrm{~m}, 4 \mathrm{H}), 3.71-3.52(\mathrm{~m}, 4 \mathrm{H}), 3.31-2.90(\mathrm{~m}, 4 \mathrm{H})$ ppm. ${ }^{13} \mathrm{C} \mathrm{NMR}\left(\mathrm{CD}_{2} \mathrm{Cl}_{2}, \delta\right), 134.8(\mathrm{~d}, J(\mathrm{P}, \mathrm{C})=11.4 \mathrm{~Hz}), 134.5(\mathrm{~d}, J(\mathrm{P}, \mathrm{C})=10.8 \mathrm{~Hz}), 132.4(\mathrm{~d}, J(\mathrm{P}, \mathrm{C})=6.6$ $\mathrm{Hz}), 131.0,129.5(\mathrm{~d}, J(\mathrm{P}, \mathrm{C})=7.4 \mathrm{~Hz}), 129.1,79.3(\mathrm{~d}, J(\mathrm{P}, \mathrm{C})=101 \mathrm{~Hz}), 79.0(\mathrm{~d}, J(\mathrm{P}, \mathrm{C})=101 \mathrm{~Hz}), 72.1(\mathrm{~d}$, $J(\mathrm{P}, \mathrm{C})=11.9 \mathrm{~Hz}), 72.0(\mathrm{~d}, J(\mathrm{P}, \mathrm{C})=12.1 \mathrm{~Hz}), 72.4(\mathrm{~d}, J(\mathrm{P}, \mathrm{C})=15.7 \mathrm{~Hz}), 72.0(\mathrm{~d}, J(\mathrm{P}, \mathrm{C})=14.9 \mathrm{~Hz}), 71.7,71.5$, 37.1, 35.4, 34.6, 34.5 ppm. ${ }^{31} \mathrm{P}$ NMR $\left(\mathrm{CD}_{2} \mathrm{Cl}_{2}, \delta\right), 79.6$ and $79.1 \mathrm{ppm}$. Mass spectrum $\left(\mathrm{EI} I^{+}, \mathrm{m} / \mathrm{z}\right), 756[\mathrm{M}]^{+}$. Accurate mass measurement $\left[\mathrm{EI}^{+}, \mathrm{m} / \mathrm{z}\right]: 755.8835[\mathrm{M}]^{+}$, calculated mass for $\mathrm{C}_{30} \mathrm{H}_{30} \mathrm{Fe}_{2} \mathrm{P}_{2} \mathrm{~S}_{6}: 755.8840$.

Compound 6. Orange solid (46\% yield). Two diastereomers were found in ca. 1:2 intensity ratio. Selected IR $\left(\mathrm{KBr}, \mathrm{cm}^{-1}\right):$ 1484(m), 1408(m), 1258(m), 1170(s), 1105(m), 1024(s), 1000(m), 951(m), 824(s), 706(s), 676(vs), 530(m), 474(s). ${ }^{1} \mathrm{H}$ NMR ( $\mathrm{CD}_{2} \mathrm{Cl}_{2}, \delta$ ), 7.66 (s, 2H), 7.22-7.16 (m, 6H), 4.58-4.55 (m, 8H), 4.47-4.43 $(\mathrm{m}, 8 \mathrm{H}), 4.30(\mathrm{~s}, 10 \mathrm{H}), 4.29(\mathrm{~s}, 10 \mathrm{H}), 4.18-3.96(\mathrm{~m}, 4 \mathrm{H}), 3.66-3.61(\mathrm{~m}, 4 \mathrm{H}), 3.15-2.66(\mathrm{~m}, 8 \mathrm{H}) \mathrm{ppm} .{ }^{13} \mathrm{C}$ NMR $\left(\mathrm{CD}_{2} \mathrm{Cl}_{2}, \delta\right), 139.2,138.9,130.5,130.3,128.4,128.3,127.2,126.7,80.2(\mathrm{~d}, J(\mathrm{P}, \mathrm{C})=103 \mathrm{~Hz}), 79.9$ (d, $J(\mathrm{P}, \mathrm{C})=103 \mathrm{~Hz}), 72.1(\mathrm{~d}, J(\mathrm{P}, \mathrm{C})=12.1 \mathrm{~Hz}), 72.0(\mathrm{~d}, J(\mathrm{P}, \mathrm{C})=11.7 \mathrm{~Hz}), 71.4(\mathrm{~d}, J(\mathrm{P}, \mathrm{C})=15.3 \mathrm{~Hz}), 71.3(\mathrm{~d}$, $J(\mathrm{P}, \mathrm{C})=14.9 \mathrm{~Hz}), 71.0,70.8,38.3,37.7,32.7,32.6 \mathrm{ppm} .{ }^{31} \mathrm{P} \mathrm{NMR}\left(\mathrm{CD}_{2} \mathrm{Cl}_{2}, \delta\right), 82.3$ and 82.2 ppm. Mass spectrum $\left(E I^{+}, \mathrm{m} / \mathrm{z}\right), 756[\mathrm{M}]^{+}$. Accurate mass measurement $\left[\mathrm{EI}^{+}, \mathrm{m} / \mathrm{z}\right]: 755.8850[\mathrm{M}]^{+}$, calculated mass for $\mathrm{C}_{30} \mathrm{H}_{30} \mathrm{Fe}_{2} \mathrm{P}_{2} \mathrm{~S}_{6}: 755.8846$.

Compound 8. Dark yellow solid (40\% yield). Two diastereomers were found in ca. 1:1 intensity ratio. Selected IR (KBr, cm $\left.{ }^{-1}\right): 1310(\mathrm{~m}), 1232(\mathrm{~m}), 1172(\mathrm{~s}), 1106(\mathrm{~m}), 1024(\mathrm{~s}), 824(\mathrm{~s}), 705(\mathrm{vs}), 673(\mathrm{vs}), 530(\mathrm{~m})$, 477(vs). ${ }^{1} \mathrm{H}$ NMR $\left(\mathrm{CD}_{2} \mathrm{Cl}_{2}, \delta\right), 7.34-7.10$ (m, 16H), 4.62-4.60 (m, 16H), 4.32 (s, 10H), 4.31 (s, 10H), 4.11$3.89(\mathrm{~m}, 8 \mathrm{H}), 3.66-3.55(\mathrm{~m}, 8 \mathrm{H}) \mathrm{ppm} .{ }^{13} \mathrm{C} \mathrm{NMR}\left(\mathrm{CD}_{2} \mathrm{Cl}_{2}, \delta\right), 137.8,137.7,130.8,130.2,129.4,128.9,128.6$, 128.3, $78.4(\mathrm{~d}, J(\mathrm{P}, \mathrm{C})=101 \mathrm{~Hz}), 78.2(\mathrm{~d}, J(\mathrm{P}, \mathrm{C})=102 \mathrm{~Hz}), 72.4,72.3,71.9,71.7,71.2,70.9,38.0,35.6 \mathrm{ppm}$. ${ }^{31} \mathrm{P}$ NMR $\left(\mathrm{CD}_{2} \mathrm{Cl}_{2}, \delta\right), 82.0$ and $81.4 \mathrm{ppm}$. Mass spectrum $\left(\mathrm{CI}^{+}, \mathrm{m} / \mathrm{z}\right), 833[\mathrm{M}+\mathrm{H}]^{+}$. Accurate mass measurement $\left[\mathrm{CI}^{+}, \mathrm{m} / \mathrm{z}\right]: 832.9233[\mathrm{M}+\mathrm{H}]^{+}$, calculated mass for $\mathrm{C}_{36} \mathrm{H}_{34} \mathrm{Fe}_{2} \mathrm{P}_{2} \mathrm{~S}_{6} \mathrm{H}: 832.9237$.

Compound 9. Golden solid (65\% yield). Four diastereomers were found in ca. 1:1:2:2 intensity ratio. Selected IR (KBr, cm $\left.{ }^{-1}\right):$ 1583(m), 1454(m), 1409(m), 1384(m), 1169(s), 1106(m), 1023(s), 1002(m), 821(s), 766(m), 674(vs), 535(m), 478(vs). ${ }^{1} \mathrm{H}$ NMR $\left(\mathrm{CD}_{2} \mathrm{Cl}_{2}, \delta\right), 8.00$ (s, 2H), 7.98 (s, 1H), 7.96 (s, 1H), 7.47-7.10 (m, 24H), 4.72-4.63 (m, 8H), 4.51-4.45 (m, 8H), $4.36(\mathrm{~s}, 5 \mathrm{H}), 4.32(\mathrm{~s}, 5 \mathrm{H}), 4.30(\mathrm{~s}, 5 \mathrm{H}), 4.25(\mathrm{~s}, 5 \mathrm{H}), 4.14-3.84$ $(\mathrm{m}, 16 \mathrm{H}), 2.28(\mathrm{~s}, 3 \mathrm{H}), 2.27(\mathrm{~s}, 3 \mathrm{H}), 2.24(\mathrm{~s}, 3 \mathrm{H}), 2.23(\mathrm{~s}, 3 \mathrm{H}) \mathrm{ppm} .{ }^{13} \mathrm{C} \mathrm{NMR}\left(\mathrm{CD}_{2} \mathrm{Cl}_{2}, \delta\right), 141.2,140.2$, 139.0, 138.5, 137.9, 137.6, 136.0, 135.9, 135.7, 135.5, 135.2, 135.1, 135.0, 131.7, 131.4, 131.3, 130.6, 130.2, 
129.0, 128.7, 128.5, 126.9, 126.8, 126.1, 126.0, $82.0(\mathrm{~d}, J(\mathrm{P}, \mathrm{C})=89.9 \mathrm{~Hz}), 81.9(\mathrm{~d}, J(\mathrm{P}, \mathrm{C})=90.5 \mathrm{~Hz}), 78.3(\mathrm{~d}$, $J(\mathrm{P}, \mathrm{C})=90.3 \mathrm{~Hz}), 78.2(\mathrm{~d}, J(\mathrm{P}, \mathrm{C})=90.4 \mathrm{~Hz}), 73.8,73.6,73.5,73.4,72.9,72.7,72.6,72.2,71.5,71.4,71.2$, 71.1, 36.3, 36.1, 34.9, 31.0, 21.5, 21.4, 21.3, $21.2\left(\mathrm{CH}_{3}\right)$ ppm. ${ }^{31} \mathrm{P}$ NMR $\left(\mathrm{CD}_{2} \mathrm{Cl}_{2}, \delta\right), 80.9,80.7,78.1$ and 78.0 ppm. Mass spectrum $\left(\mathrm{CI}^{+}, \mathrm{m} / \mathrm{z}\right), 819[\mathrm{M}+\mathrm{H}]^{+}$. Accurate mass measurement $\left[\mathrm{CI}^{+}, \mathrm{m} / \mathrm{z}\right]: 818.9080[\mathrm{M}+\mathrm{H}]^{+}$, calculated mass for $\mathrm{C}_{35} \mathrm{H}_{32} \mathrm{Fe}_{2} \mathrm{P}_{2} \mathrm{~S}_{6} \mathrm{H}: 818.9081$.

Compound 10a. Yellow paste $(0.250 \mathrm{~g}, 33 \%)$. Selected IR (KBr, $\left.\mathrm{cm}^{-1}\right): 1410(\mathrm{~m}), 1300(\mathrm{~m}), 1252(\mathrm{~m}), 1173(\mathrm{~s})$, 1106(m), 1024(s), 1002(m), 825(s), 767(m), 732(m), 675(vs), 615(m), 535(s), 483(vs). ${ }^{1} \mathrm{H}$ NMR $\left(\mathrm{CDCl}_{3}, \delta\right)$, 7.32-7.10 (m, 4H), 4.83-4.53 (m, 8H), 4.43 (s, 5H), 4.41 (s, 5H), 4.19-4.13 (m, 4H), 3.32-3.10 (m, 4H), 2.29$2.21(\mathrm{~m}, 2 \mathrm{H}) \mathrm{ppm} .{ }^{13} \mathrm{C} \mathrm{NMR}\left(\mathrm{CDCl}_{3}, \delta\right), 136.3(\mathrm{~d}, J(\mathrm{P}, \mathrm{C})=66.7 \mathrm{~Hz}), 134.1(\mathrm{~d}, J(\mathrm{P}, \mathrm{C})=10.4 \mathrm{~Hz}), 132.8$, $131.2(\mathrm{~d}, J(\mathrm{P}, \mathrm{C})=11.7 \mathrm{~Hz}), 127.9,126.8,78.3(\mathrm{~d}, J(\mathrm{P}, \mathrm{C})=100.9 \mathrm{~Hz}), 72.1(\mathrm{~d}, J(\mathrm{P}, \mathrm{C})=12.0 \mathrm{~Hz}), 71.4(\mathrm{~d}$, $J(\mathrm{P}, \mathrm{C})=15.0 \mathrm{~Hz}), 71.0,35.8,32.1,29.7 \mathrm{ppm} .{ }^{31} \mathrm{P} \mathrm{NMR}\left(\mathrm{CDCl}_{3}, \delta\right), 79.4 \mathrm{ppm}$. Mass spectrum $(\mathrm{EI}, \mathrm{m} / \mathrm{z}), 770$ $[\mathrm{M}]^{+}$. Accurate mass measurement $\left[\mathrm{EI}^{+}, \mathrm{m} / \mathrm{z}\right]: 769.9004[\mathrm{M}]^{+}$, calculated mass for $\mathrm{C}_{31} \mathrm{H}_{32} \mathrm{Fe}_{2} \mathrm{P}_{2} \mathrm{~S}_{6}: 769.9002$.

Compound 10b. Dark yellow paste (0.220 g, 29\%). Selected IR (KBr, cm $\left.{ }^{-1}\right)$ : 1409(m), 1298(m), 1244(m), 1170(s), 1106(m), 1024(s), 1002(m), 908(s), 824(s), 768(m), 731(s), 674(s), 618(m), 532(s), 478(vs). ${ }^{1} \mathrm{H}$ NMR $\left(\mathrm{CDCl}_{3}, \delta\right), 7.35-7.27(\mathrm{~m}, 4 \mathrm{H}), 4.79-4.56(\mathrm{~m}, 8 \mathrm{H}), 4.42(\mathrm{~s}, 10 \mathrm{H}), 4.16-4.10(\mathrm{~m}, 4 \mathrm{H}), 3.26-3.07(\mathrm{~m}, 4 \mathrm{H}), 2.24-$ $2.20(\mathrm{~m}, 2 \mathrm{H}) \mathrm{ppm} .{ }^{13} \mathrm{C} \mathrm{NMR}\left(\mathrm{CDCl}_{3}, \delta\right), 140.1,134.1(\mathrm{~d}, J(\mathrm{P}, \mathrm{C})=3.1 \mathrm{~Hz}), 131.6,128.8,78.5(\mathrm{~d}, J(\mathrm{P}, \mathrm{C})=$ $100.5 \mathrm{~Hz}), 72.0(\mathrm{~d}, J(\mathrm{P}, \mathrm{C})=12.0 \mathrm{~Hz}), 71.5(\mathrm{~d}, J(\mathrm{P}, \mathrm{C})=15.1 \mathrm{~Hz}), 71.0,35.7,32.5,29.4 \mathrm{ppm} .{ }^{31} \mathrm{P}$ NMR $\left(\mathrm{CDCl}_{3}, \delta\right), 79.5$ ppm. Mass spectrum $\left(\mathrm{EI}^{+}, \mathrm{m} / \mathrm{z}\right), 770[\mathrm{M}]^{+}$. Accurate mass measurement $\left[\mathrm{EI}^{+}, \mathrm{m} / \mathrm{z}\right]: 769.9004$ $[\mathrm{M}]^{+}$, calculated mass for $\mathrm{C}_{31} \mathrm{H}_{32} \mathrm{Fe}_{2} \mathrm{P}_{2} \mathrm{~S}_{6}: 769.9002$.

Compound 11. Dark yellow paste (0.402 g, 51\%). Selected IR (KBr, cm $\left.{ }^{-1}\right): 1455(\mathrm{~m}), 1414(\mathrm{~m}), 1172(\mathrm{~s})$, 1105(m), 1024(s), 1002(m), 971(m), 908(m), 824(s), 765(m), 731(m), 674(s), 530(m), 479(s). ${ }^{1} \mathrm{H}$ NMR $\left(\mathrm{CDCl}_{3}, \delta\right), 7.42-7.19(\mathrm{~m}, 4 \mathrm{H}), 4.68-4.50(\mathrm{~m}, 8 \mathrm{H}), 4.39(\mathrm{~s}, 10 \mathrm{H}), 3.99-3.84(\mathrm{~m}, 4 \mathrm{H}), 3.43-3.39(\mathrm{~m}, 4 \mathrm{H}), 1.97-$ $1.84(\mathrm{~m}, 4 \mathrm{H})$ ppm. ${ }^{13} \mathrm{C} \mathrm{NMR}\left(\mathrm{CDCl}_{3}, \delta\right), 135.4,131.2,128.3,80.1(\mathrm{~d}, J(\mathrm{P}, \mathrm{C})=96.0 \mathrm{~Hz}), 71.8(\mathrm{~d}, J(\mathrm{P}, \mathrm{C})=$ $11.8 \mathrm{~Hz}), 71.4(\mathrm{~d}, J(\mathrm{P}, \mathrm{C})=14.9 \mathrm{~Hz}), 70.8,35.2,32.9,28.6 \mathrm{ppm} .{ }^{31} \mathrm{P} \mathrm{NMR}\left(\mathrm{CDCl}_{3}, \delta\right), 79.5 \mathrm{ppm}$. Mass spectrum $\left(\mathrm{EI}^{+}, \mathrm{m} / \mathrm{z}\right), 784[\mathrm{M}]^{+}$. Accurate mass measurement $\left[\mathrm{EI}^{+}, \mathrm{m} / \mathrm{z}\right]: 783.9156[\mathrm{M}]^{+}$, calculated mass for $\mathrm{C}_{32} \mathrm{H}_{34} \mathrm{Fe}_{2} \mathrm{P}_{2} \mathrm{~S}_{6}: 783.9159$.

Compound 12a. Pale yellow paste (0.200 g, 25\%). Selected IR (KBr, cm $\left.{ }^{-1}\right)$ : 1489(m), 1453(m), 1410(m), 1171(s), 1106(m), 1024(s), 908(m), 824(s), 766(m), 731(s), 676(vs), 618(m), 530(s), 479(vs), 259(s). ${ }^{1} \mathrm{H}$ NMR $\left(\mathrm{CDCl}_{3}, \delta\right), 7.32-7.17(\mathrm{~m}, 4 \mathrm{H}), 4.73-4.50(\mathrm{~m}, 8 \mathrm{H}), 4.39(\mathrm{~s}, 10 \mathrm{H}), 4.36-4.16(\mathrm{~m}, 4 \mathrm{H}), 3.94-3.83(\mathrm{~m}, 4 \mathrm{H}), 3.23-$ $2.97(\mathrm{~m}, 4 \mathrm{H}), 1.90-1.55(\mathrm{~m}, 4 \mathrm{H}) \mathrm{ppm} .{ }^{13} \mathrm{C} \mathrm{NMR}\left(\mathrm{CDCl}_{3}, \delta\right), 138.7,135.0(\mathrm{~d}, J(\mathrm{P}, \mathrm{C})=3.1 \mathrm{~Hz}), 130.9,128.3$, $79.7(\mathrm{~d}, J(\mathrm{P}, \mathrm{C})=100.9 \mathrm{~Hz}), 71.8(\mathrm{~d}, J(\mathrm{P}, \mathrm{C})=12.5 \mathrm{~Hz}), 71.4(\mathrm{~d}, J(\mathrm{P}, \mathrm{C})=15.5 \mathrm{~Hz}), 70.8,35.5,33.2,28.8,26.9$ 
ppm. ${ }^{31} \mathrm{P} \mathrm{NMR}\left(\mathrm{CDCl}_{3}, \delta\right), 81.8 \mathrm{ppm}$. Mass spectrum $\left(\mathrm{EI}^{+}, \mathrm{m} / \mathrm{z}\right), 812[\mathrm{M}]^{+}$. Accurate mass measurement $\left[\mathrm{EI}^{+}\right.$, $\mathrm{m} / \mathrm{z}]: 811.9468[\mathrm{M}]^{+}$, calculated mass for $\mathrm{C}_{34} \mathrm{H}_{38} \mathrm{Fe}_{2} \mathrm{P}_{2} \mathrm{~S}_{6}: 811.9471$.

Compound 12b. Pale yellow paste $(0.250 \mathrm{~g}, 31 \%)$. Two diastereomers were found in $c a .3: 2$ intensity ratio. Selected IR (KBr, cm $\left.{ }^{-1}\right)$ : 1489(m), 1453(m), 1410(m), 1385(m), 1170(s), 1106(m), 1024(s), 1001(m), 823(s), 765(m), 676(vs), 618(m), 529(m), 478(vs), 259(s). ${ }^{1} \mathrm{H}$ NMR $\left(\mathrm{CDCl}_{3}, \delta\right), 7.37-7.19(\mathrm{~m}, 8 \mathrm{H}), 4.75-4.52(\mathrm{~m}$, $16 \mathrm{H}), 4.40(\mathrm{~s}, 10 \mathrm{H}), 4.39(\mathrm{~s}, 10 \mathrm{H}), 4.36-4.17(\mathrm{~m}, 8 \mathrm{H}), 3.93-3.84(\mathrm{~m}, 8 \mathrm{H}), 3.21-2.90(\mathrm{~m}, 8 \mathrm{H}), 1.94-1.29(\mathrm{~m}, 8 \mathrm{H})$ ppm. ${ }^{13} \mathrm{C} \mathrm{NMR}\left(\mathrm{CDCl}_{3}, \delta\right), 140.2,135.2(\mathrm{~d}, J(\mathrm{P}, \mathrm{C})=3.1 \mathrm{~Hz}), 133.7(\mathrm{~d}, J(\mathrm{P}, \mathrm{C})=3.1 \mathrm{~Hz}), 130.9,129.8,128.5$, 127.7, $80.1(\mathrm{~d}, J(\mathrm{P}, \mathrm{C})=100.6 \mathrm{~Hz}), 79.8(\mathrm{~d}, J(\mathrm{P}, \mathrm{C})=101.1 \mathrm{~Hz}), 71.9,71.7,71.5,71.3,70.9,70.8,35.5,35.2$, 33.6, 33.5, 29.9, 28.8, 27.0, $26.1 \mathrm{ppm} .{ }^{31} \mathrm{P} \mathrm{NMR}\left(\mathrm{CDCl}_{3}, \delta\right), 81.7$ and $80.5 \mathrm{ppm}$. Mass spectrum $\left(\mathrm{EI}^{+}, \mathrm{m} / \mathrm{z}\right)$, $812[\mathrm{M}]^{+}$. Accurate mass measurement $\left[\mathrm{EI}^{+}, \mathrm{m} / \mathrm{z}\right]: 811.9467[\mathrm{M}]^{+}$, calculated mass for $\mathrm{C}_{34} \mathrm{H}_{38} \mathrm{Fe}_{2} \mathrm{P}_{2} \mathrm{~S}_{6}$ : 811.9471 .

Compound 14. Pale-yellow solid (0.080 g, 10\%). Selected IR (KBr, cm $\left.{ }^{-1}\right)$ : 1510(m), 1407(m), 1242(m), 1196(s), 1150(m), 1104(m), 1023(s), 891(m), 844(m), 810(s), 768(m), 656(m), 632(m), 560(s), 501(s), 477(s). ${ }^{1} \mathrm{H}$ NMR $\left(\mathrm{CDCl}_{3}, \delta\right), 7.34-7.18(\mathrm{~m}, 8 \mathrm{H}), 4.68-4.54(\mathrm{~m}, 8 \mathrm{H}), 4.40$ (s, 10H), 4.38-4.34 (m, 4H), 3.91-3.84 (m, 4H) ppm. ${ }^{13} \mathrm{C} \mathrm{NMR}\left(\mathrm{CDCl}_{3}, \delta\right), 136.8,129.1,78.5(\mathrm{~d}, J(\mathrm{P}, \mathrm{C})=92.6 \mathrm{~Hz}), 72.1(\mathrm{~d}, J(\mathrm{P}, \mathrm{C})=12.5 \mathrm{~Hz}), 71.8(\mathrm{~d}$, $J(\mathrm{P}, \mathrm{C})=15.6 \mathrm{~Hz}), 70.5,35.0$ ppm. ${ }^{31} \mathrm{P} \mathrm{NMR}\left(\mathrm{CDCl}_{3}, \delta\right), 59.8 \mathrm{ppm}$. Mass spectrum $(\mathrm{CI}+\mathrm{m} / \mathrm{z}), 801[\mathrm{M}+\mathrm{H}]^{+}$. Accurate mass measurement $\left[\mathrm{CI}^{+}, \mathrm{m} / \mathrm{z}\right]: 800.9690[\mathrm{M}+\mathrm{H}]^{+}$, calculated mass for $\mathrm{C}_{36} \mathrm{H}_{34} \mathrm{Fe}_{2} \mathrm{O}_{2} \mathrm{~S}_{4} \mathrm{H}: 800.9694$. 
3. Details of the X-ray Data Collections and Refinements for 10a, 10b, 11, 12a, $12 \mathrm{~b}$ and 14

Table S1. Details of the X-ray data collections and refinements for $\mathbf{1 0 a}, \mathbf{1 0 b}$ and $\mathbf{1 1}$

\begin{tabular}{llll}
\hline Compound & $\mathbf{1 0 a}$ & $\mathbf{1 0 b}$ & $\mathbf{1 1}$ \\
Formula & $\mathrm{C}_{31} \mathrm{H}_{32} \mathrm{Fe}_{2} \mathrm{P}_{2} \mathrm{~S}_{6}$ & $\mathrm{C}_{31} \mathrm{H}_{32} \mathrm{Fe}_{2} \mathrm{P}_{2} \mathrm{~S}_{6}$ & $\mathrm{C}_{32} \mathrm{H}_{34} \mathrm{Fe}_{2} \mathrm{P}_{2} \mathrm{~S}_{6}$ \\
$M$ & 770.60 & 770.60 & 784.62 \\
Crystal system & monoclinic & monoclinic & monoclinic \\
Space group & $C 2 / c$ & $C c$ & $P 2{ }_{1} / c$ \\
$a / \AA$ & $8.3844(16)$ & $12.3542(13)$ & $17.762(3)$ \\
$b / \AA$ & $12.371(2)$ & $8.3008(9)$ & $12.2131(17)$ \\
$c / \AA$ & $30.530(5)$ & $31.047(3)$ & $16.275(2)$ \\
$A$ & 90 & 90 & 90 \\
$B$ & $92.731(5)$ & $93.806(3)$ & $115.073(3)$ \\
$\Gamma$ & 90 & 90 & 90 \\
$U / A^{3}$ & $3163.1(9)$ & $3176.8(6)$ & $3197.8(8)$ \\
$Z$ & 4 & 4 & 4 \\
$\mu / c m^{-1}$ & 14.366 & 14.303 & 14.225 \\
Reflections collected & 17845 & 31053 & 38576 \\
Independent reflections & 2681 & 5736 & 5859 \\
$R_{\text {int }}$ & 0.0527 & 0.0486 & 0.0381 \\
$R I$ & 0.0939 & 0.0482 & 0.0393 \\
$w R 2[I>2 \sigma(I)]$ & 0.2339 & 0.1234 & 0.0995 \\
\hline & & & \\
\hline
\end{tabular}


Table S2. Details of the X-ray data collections and refinements for $\mathbf{1 2 a}, \mathbf{1 2 b}$ and $\mathbf{1 4}$

\begin{tabular}{llll}
\hline Compound & $\mathbf{1 2 a}$ & $\mathbf{1 2 b}$ & $\mathbf{1 4}$ \\
Formula & $\mathrm{C}_{34} \mathrm{H}_{38} \mathrm{Fe}_{2} \mathrm{P}_{2} \mathrm{~S}_{6}$ & $\mathrm{C}_{34} \mathrm{H}_{38} \mathrm{Fe}_{2} \mathrm{P}_{2} \mathrm{~S}_{6}$ & $\mathrm{C}_{36} \mathrm{H}_{34} \mathrm{Fe}_{2} \mathrm{O}_{2} \mathrm{P}_{2} \mathrm{~S}_{4}$ \\
$M$ & 812.68 & 812.68 & 800.54 \\
Crystal system & monoclinic & monoclinic & monoclinic \\
Space group & $P 2{ }_{1} / c$ & $C 2$ & $P 2{ }_{1} / c$ \\
$a / A$ & $7.5941(7)$ & $27.688(4)$ & $19.112(6)$ \\
$b / \AA$ & $24.3854(18)$ & $7.5726(8)$ & $11.516(4)$ \\
$c / \AA$ & $19.0840(19)$ & $34.772(6)$ & $7.491(3)$ \\
$A$ & 90 & 90 & 90 \\
$B$ & $91.056(3)$ & $106.834(4)$ & $95.025(8)$ \\
$\Gamma$ & 90 & 90 & 90 \\
$U / A^{3}$ & $3533.5(5)$ & $6978.2(17)$ & $1624.4(10)$ \\
$Z$ & 4 & 8 & 2 \\
$\mu / c m^{-1}$ & 12.903 & 13.067 & 12.689 \\
Reflections collected & 43237 & 42175 & 30856 \\
Independent reflections & 6472 & 12617 & 2989 \\
$R_{\text {int }}$ & 0.0332 & 0.0785 & 0.1499 \\
$R I$ & 0.0435 & 0.0480 & 0.0853 \\
$w R 2[I>2 \sigma(I)]$ & 0.1080 & 0.0890 & 0.2371 \\
\hline & & &
\end{tabular}




\section{4. ${ }^{1} \mathrm{H}$ and ${ }^{13} \mathrm{C}$ NMR Spectra of Compounds 1-3, 5, 6, 8, 9, 10a, 10b, 11, 12a, $12 \mathrm{~b}$ and 14}

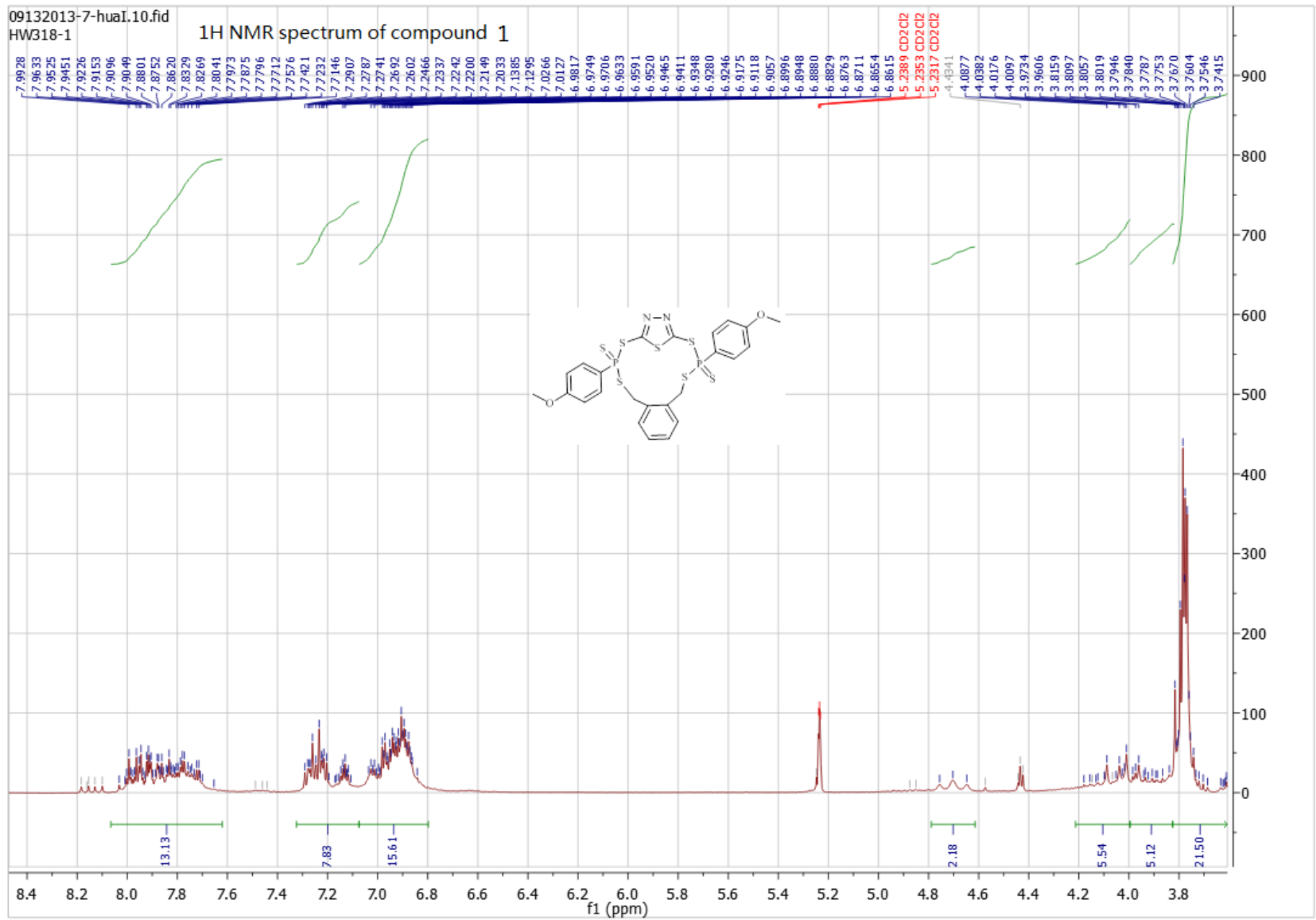




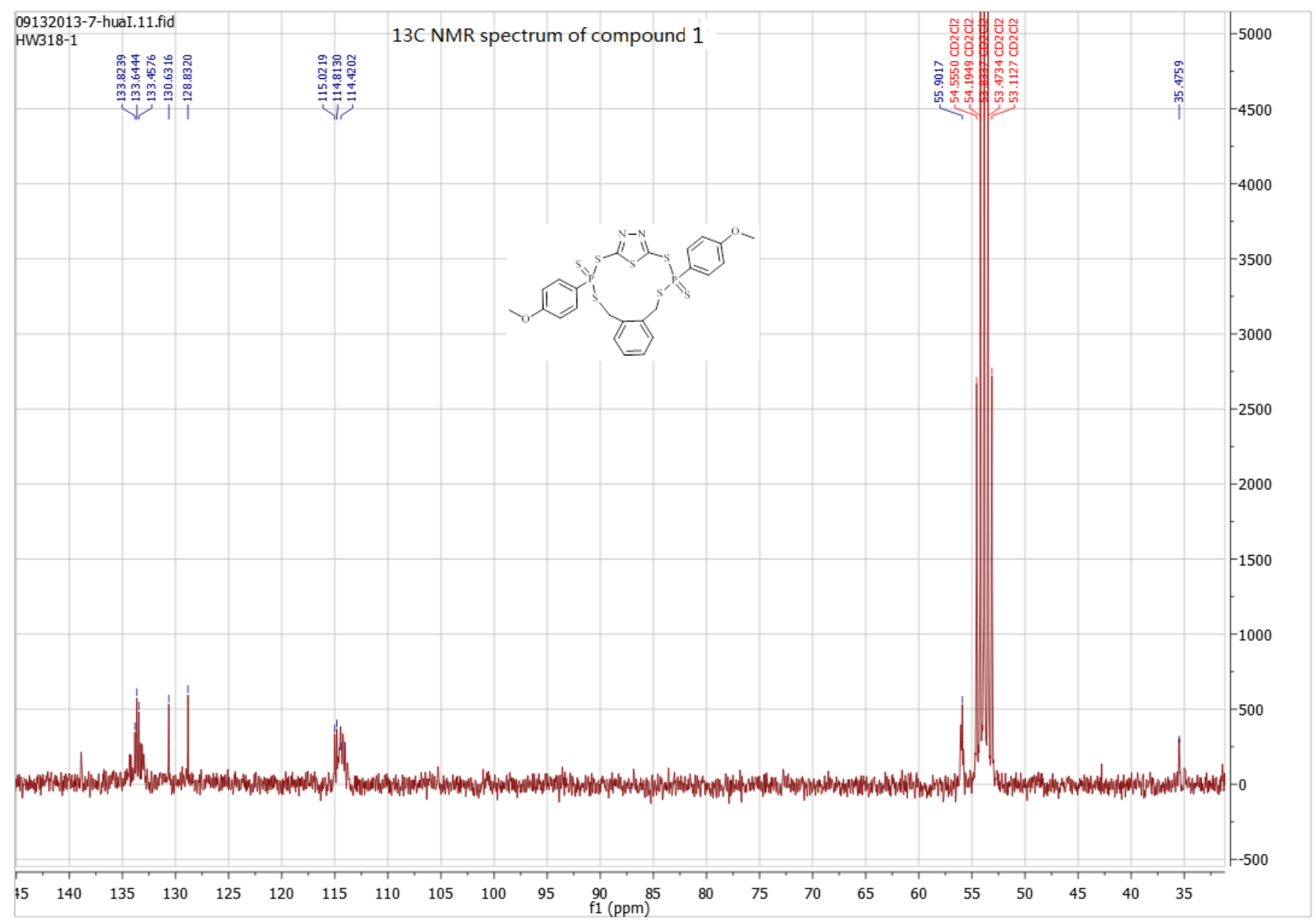




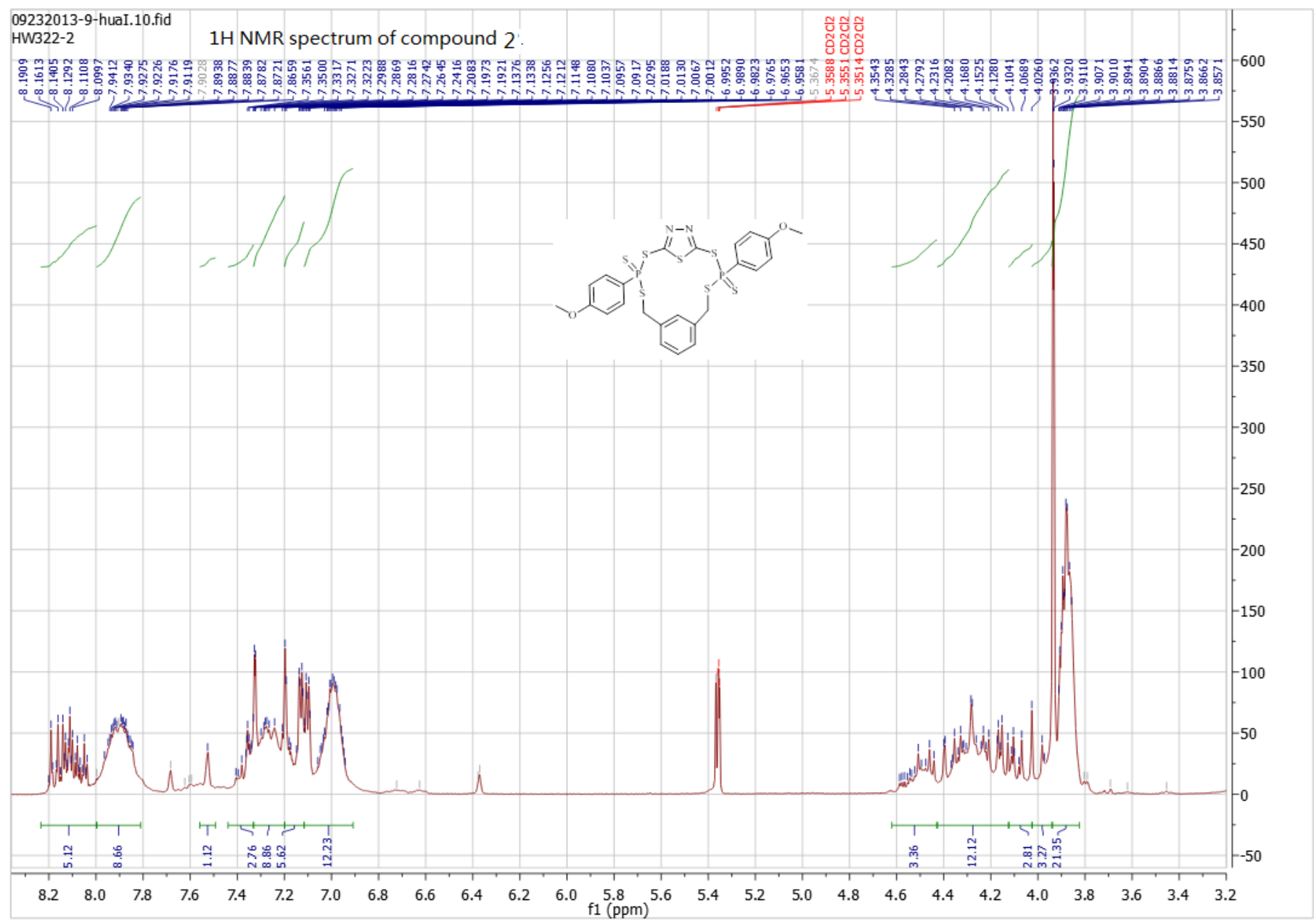




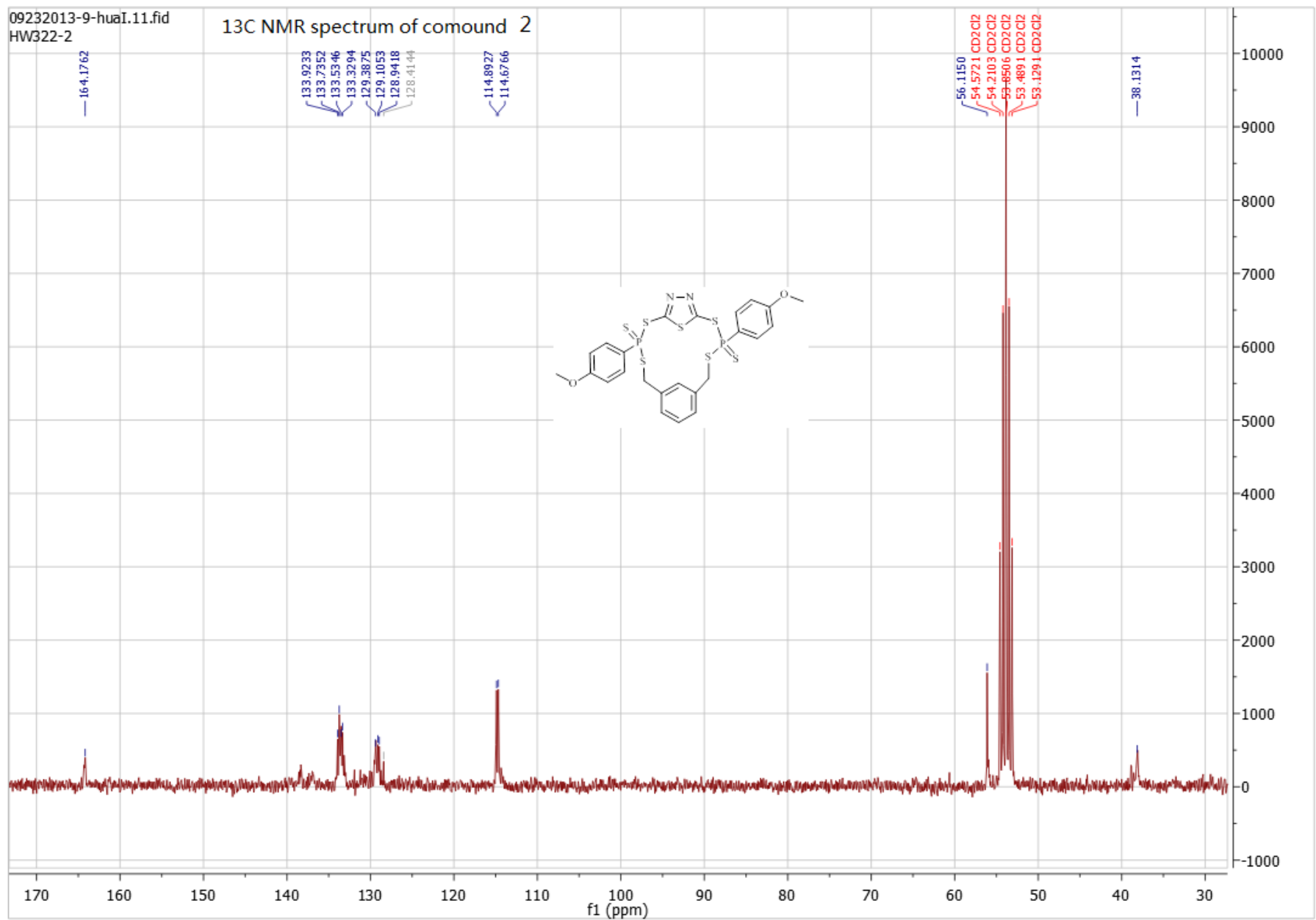




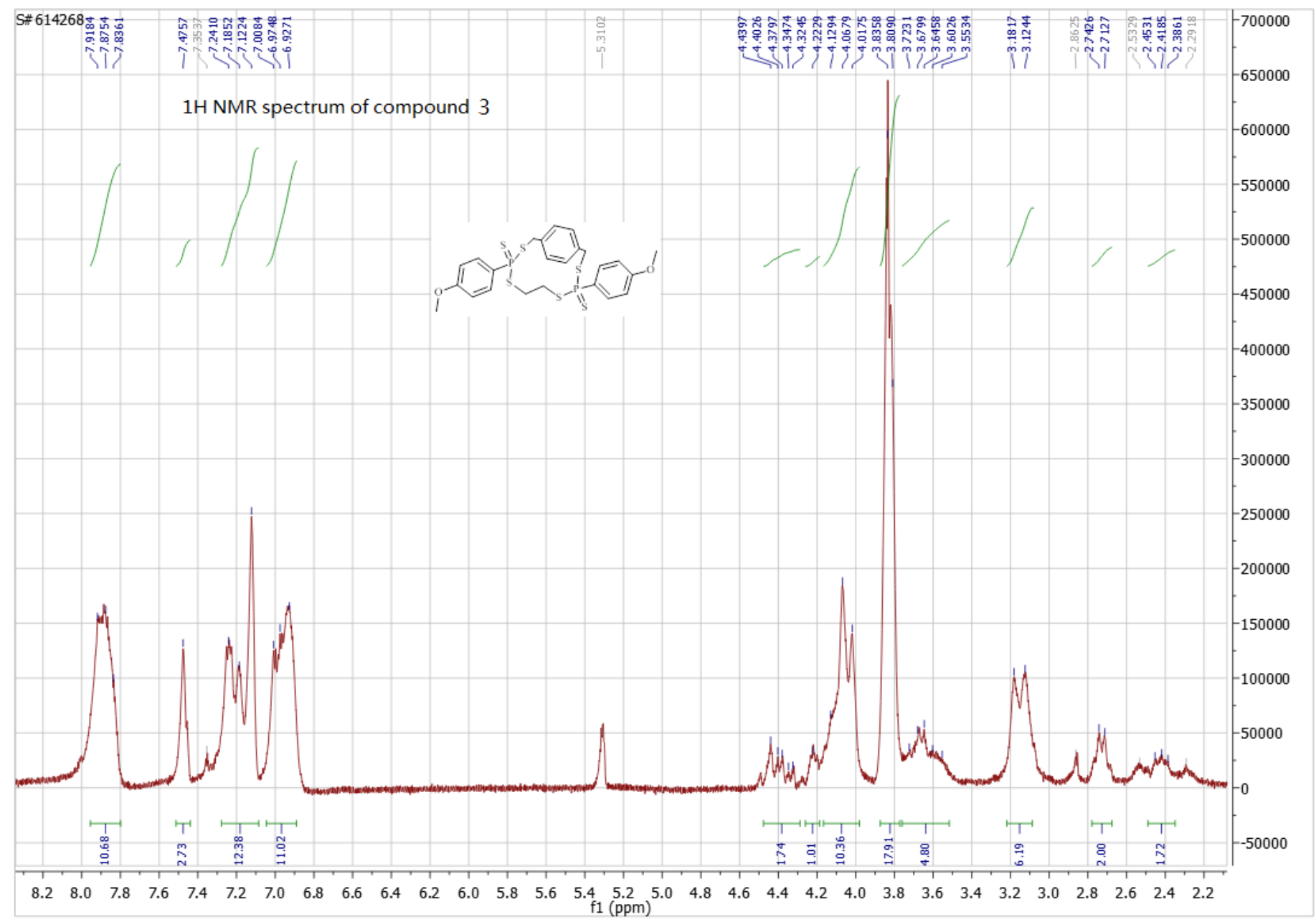




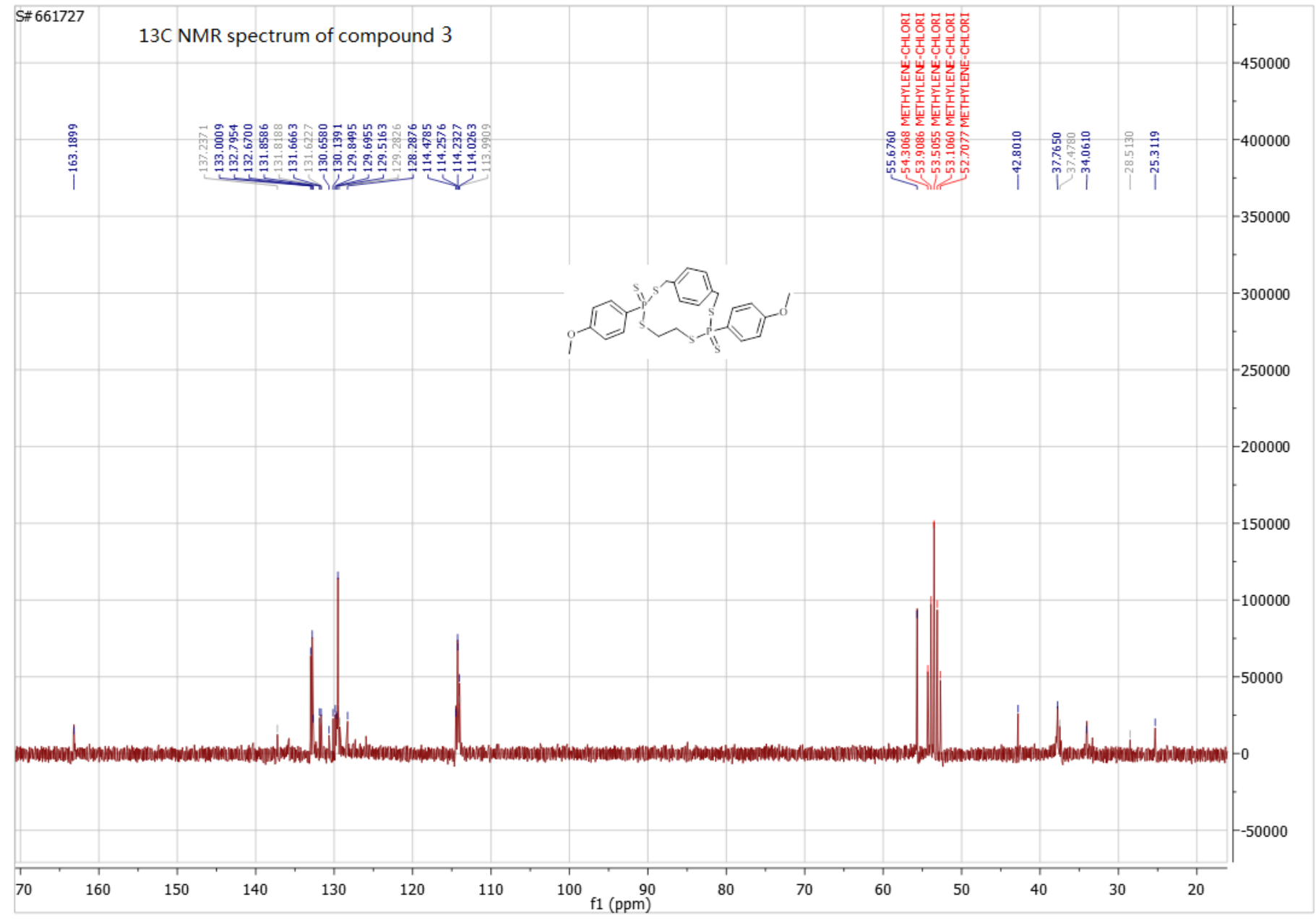




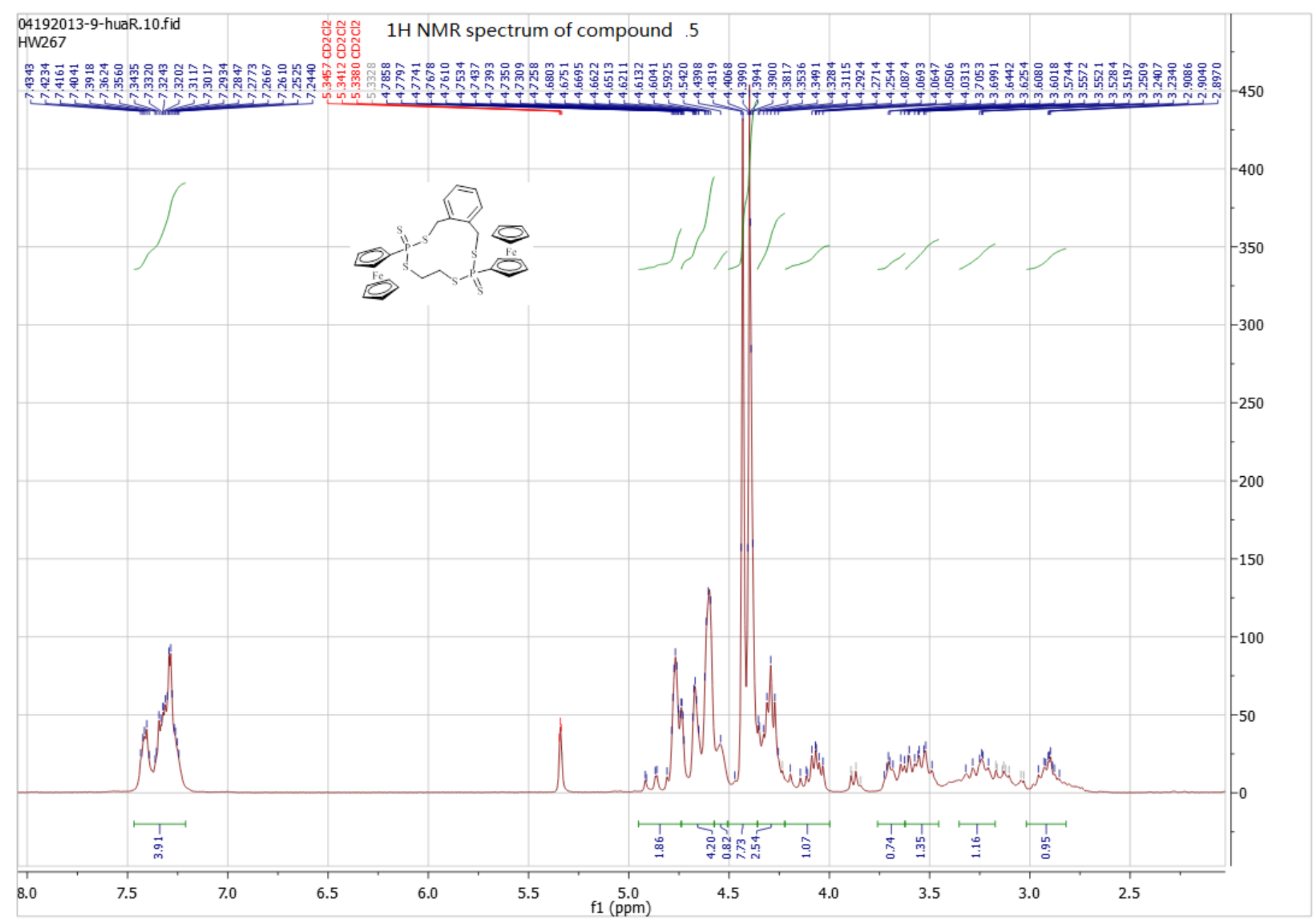




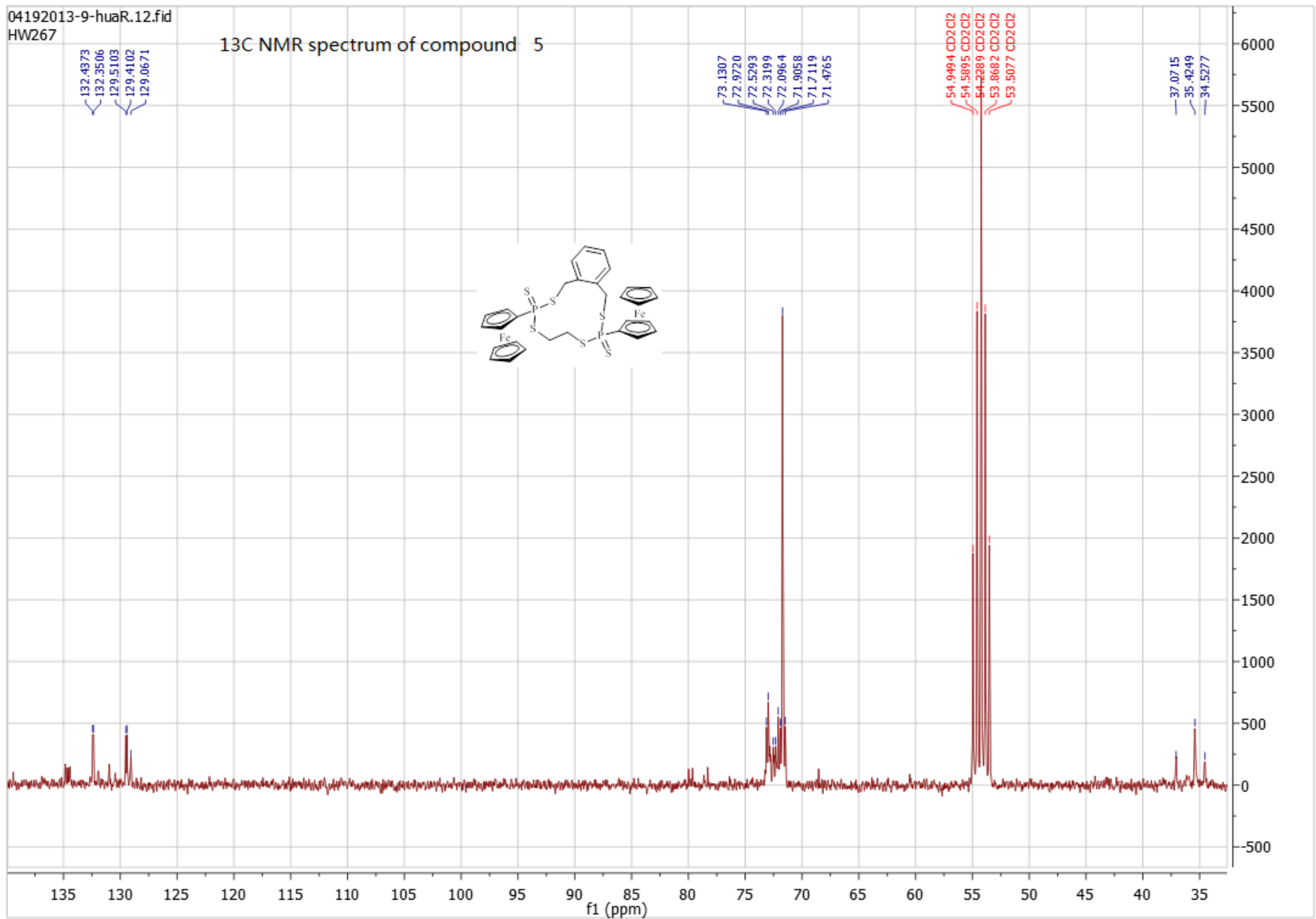




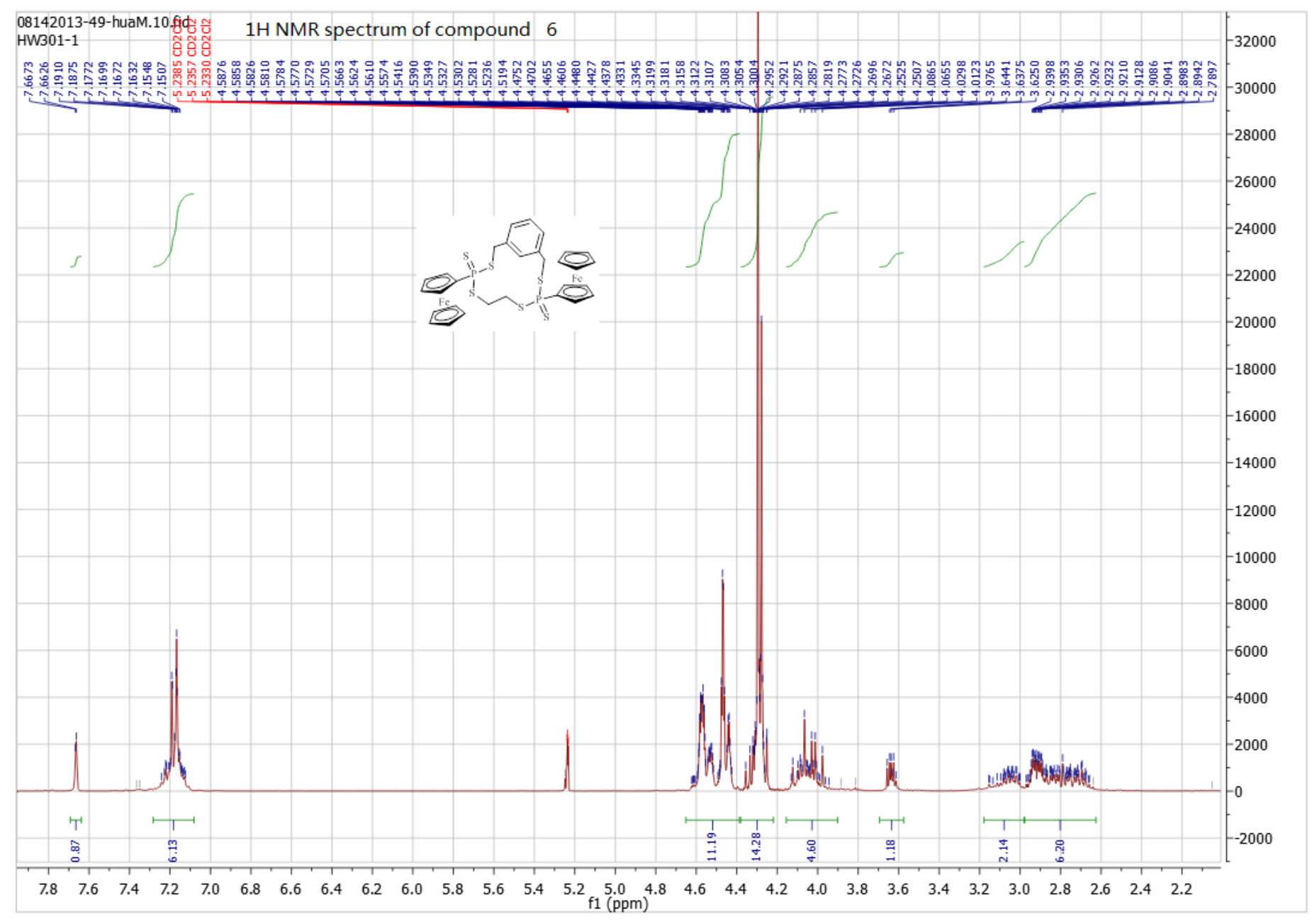




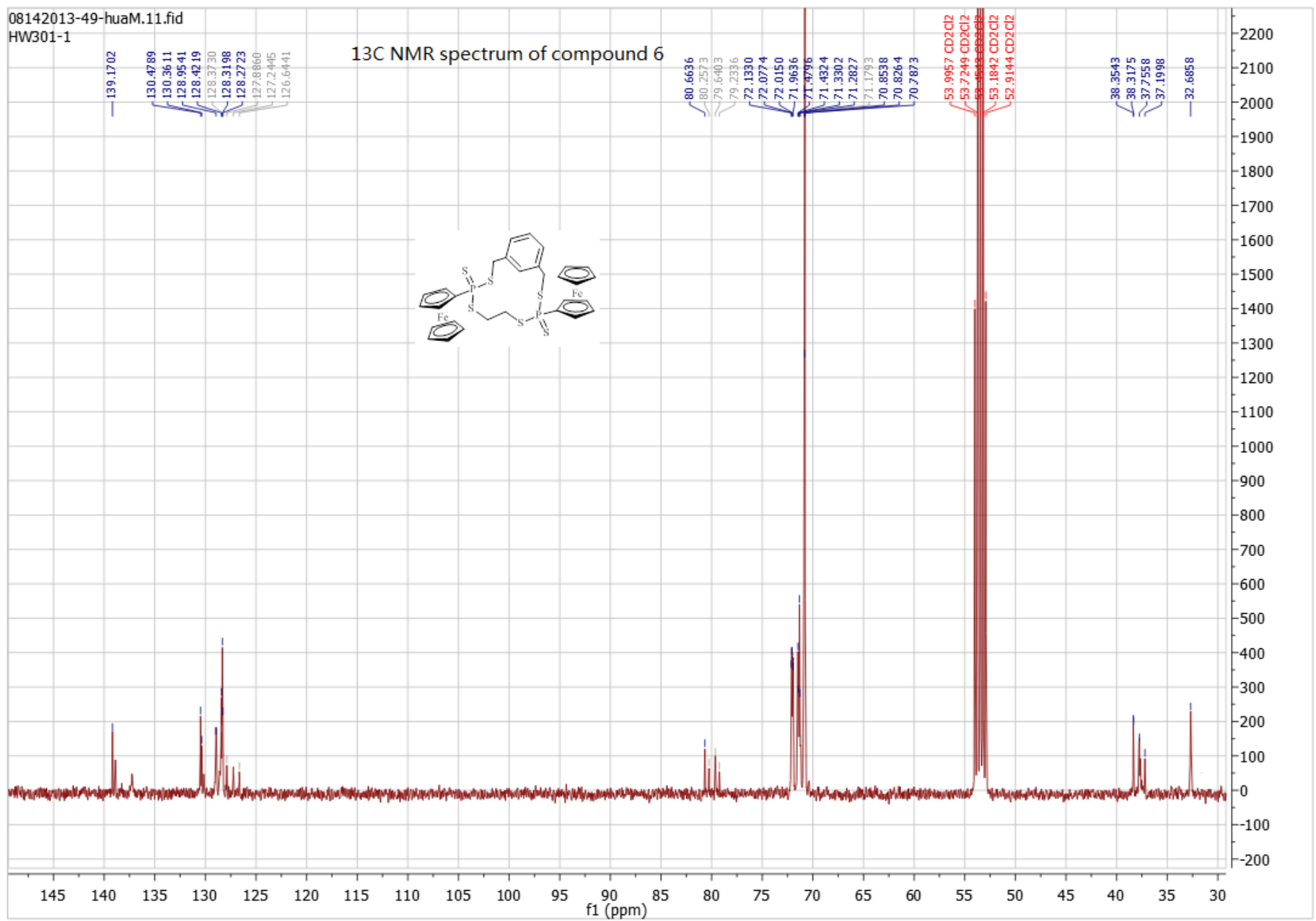




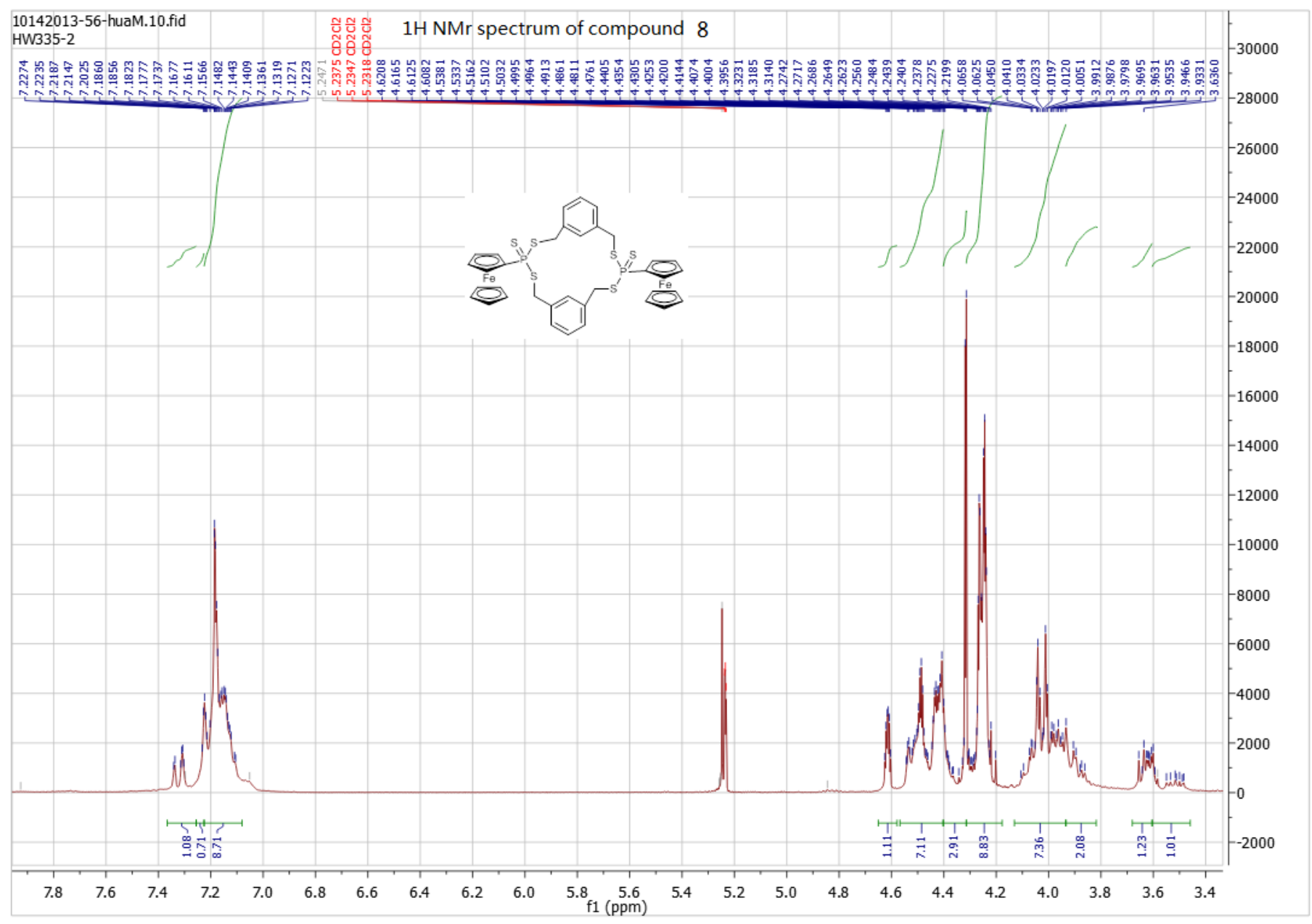




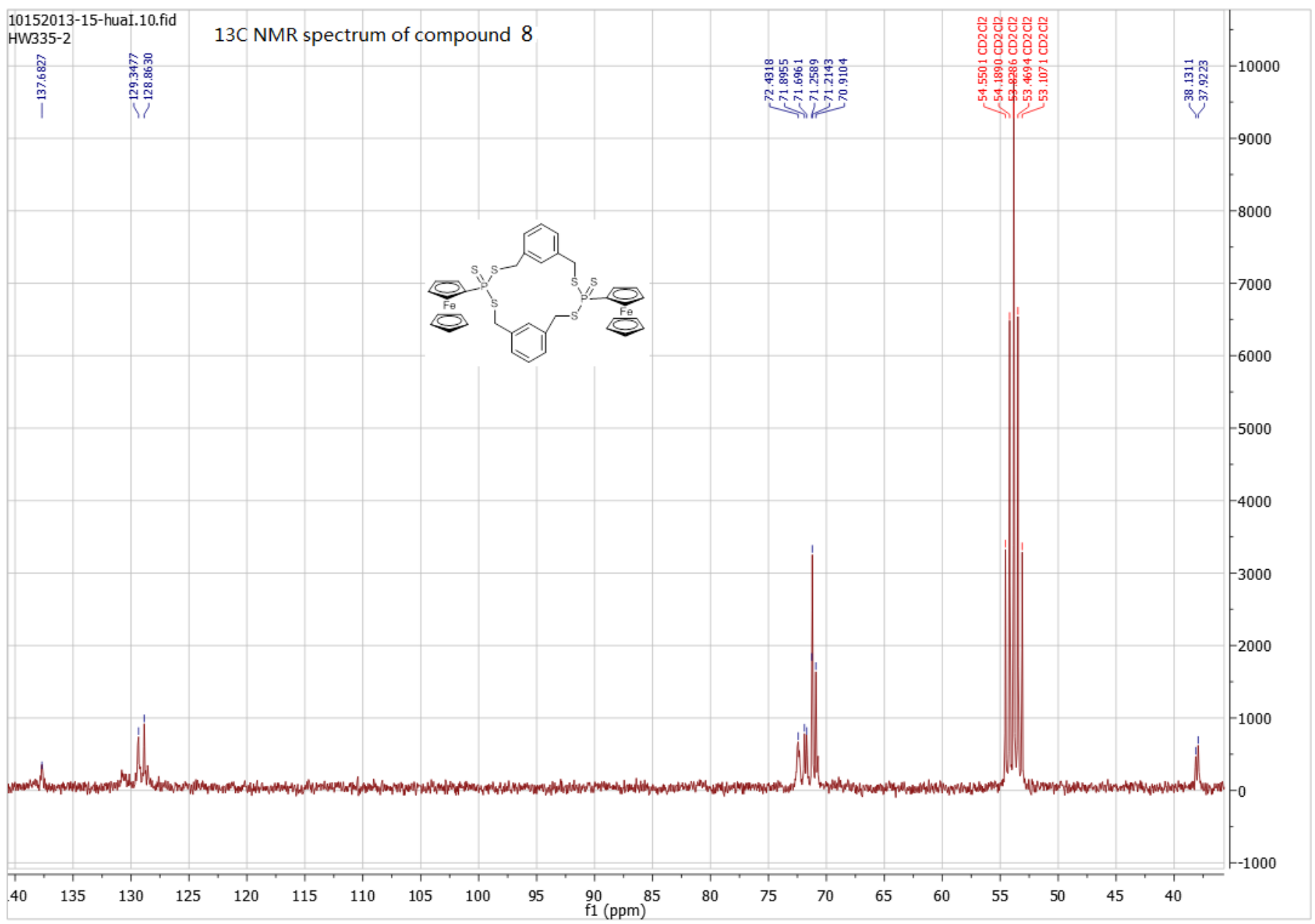




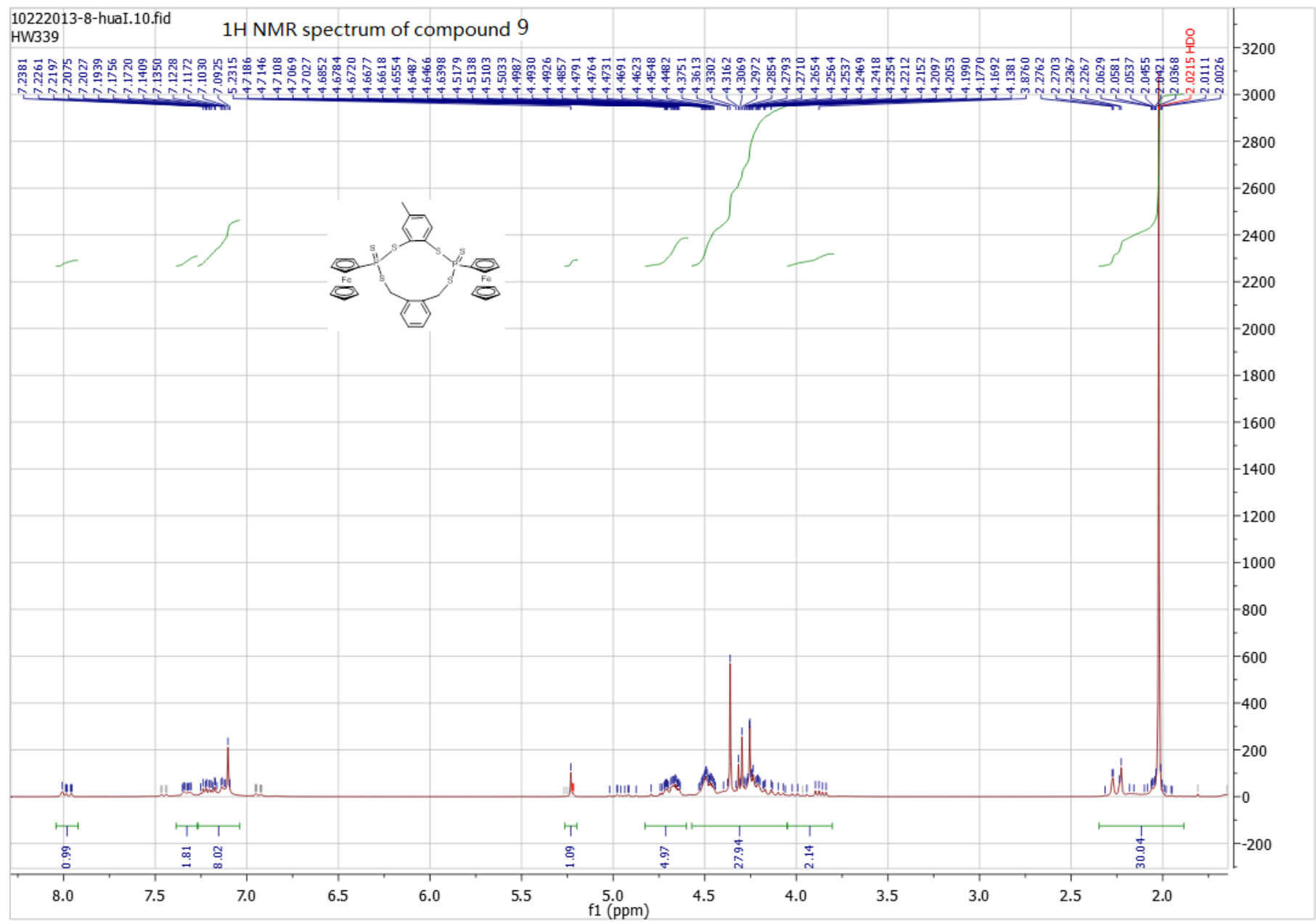




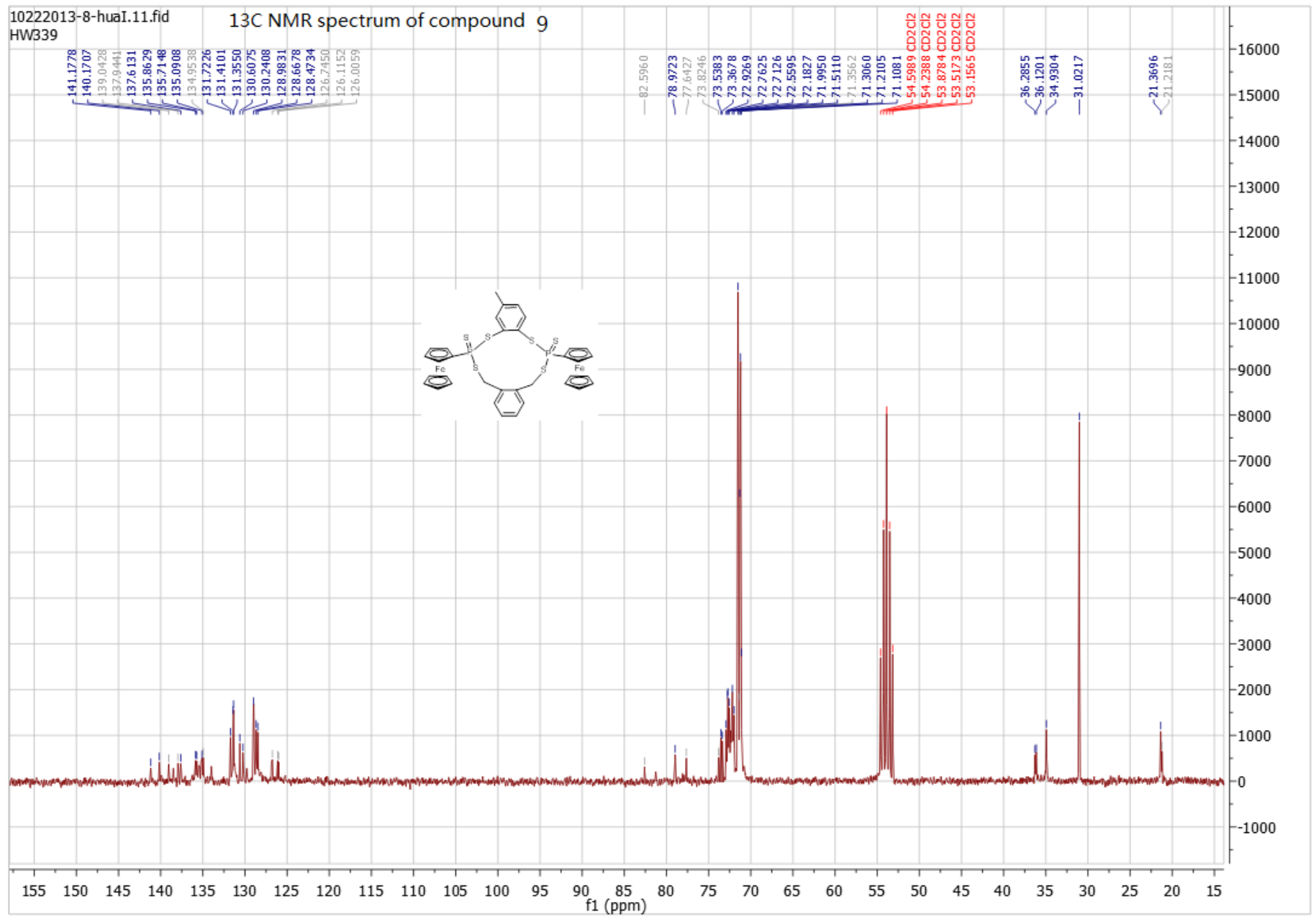




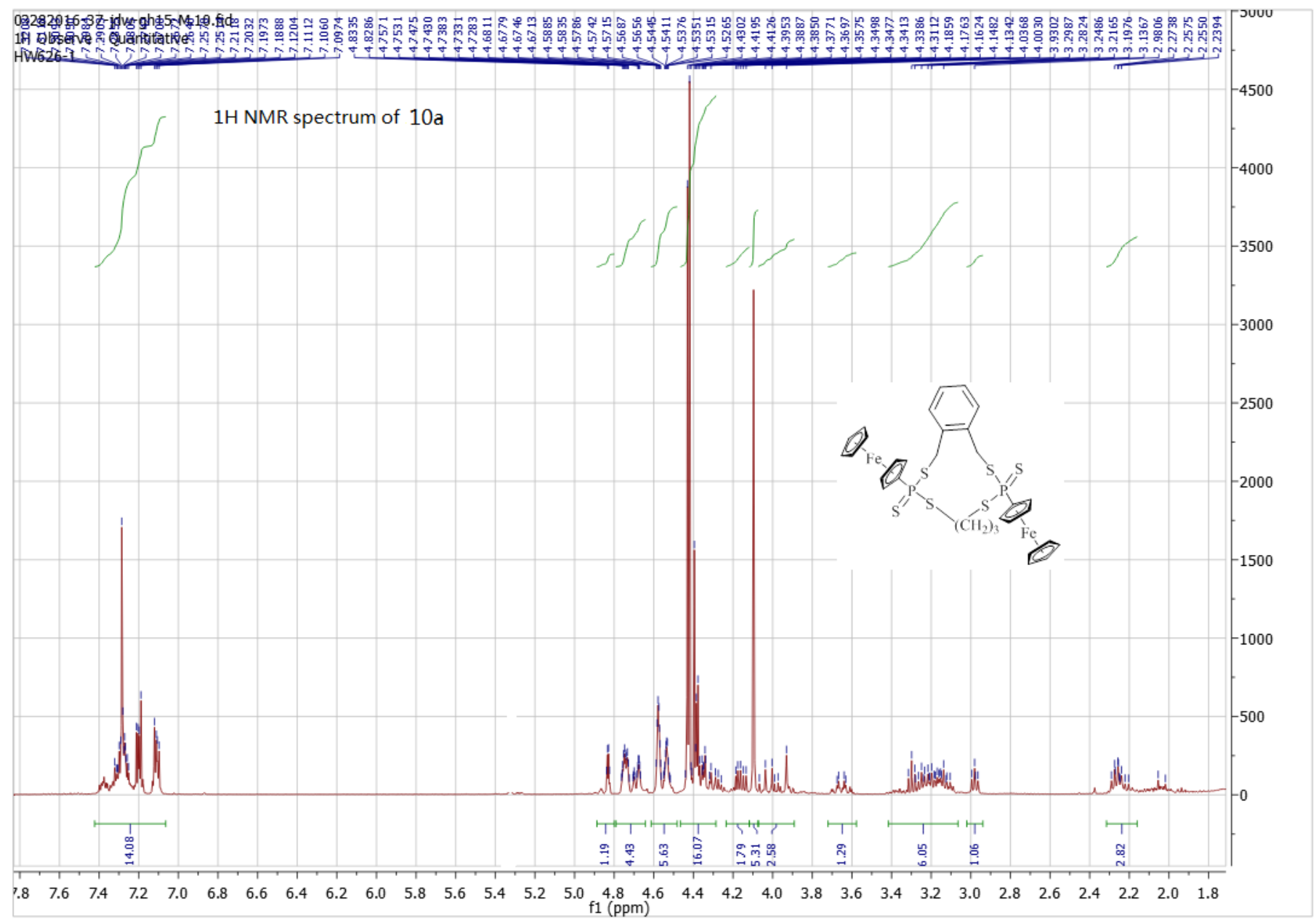




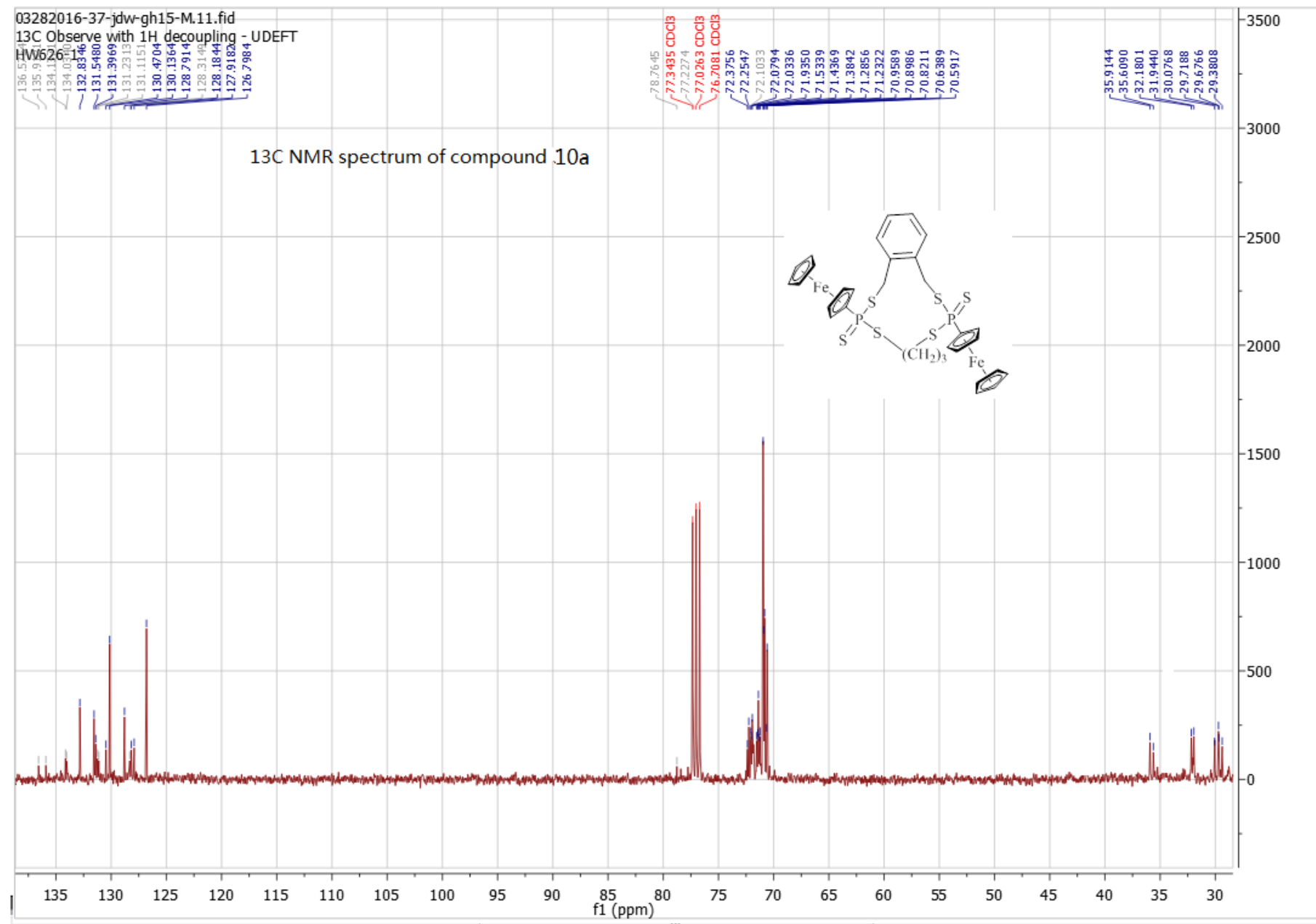




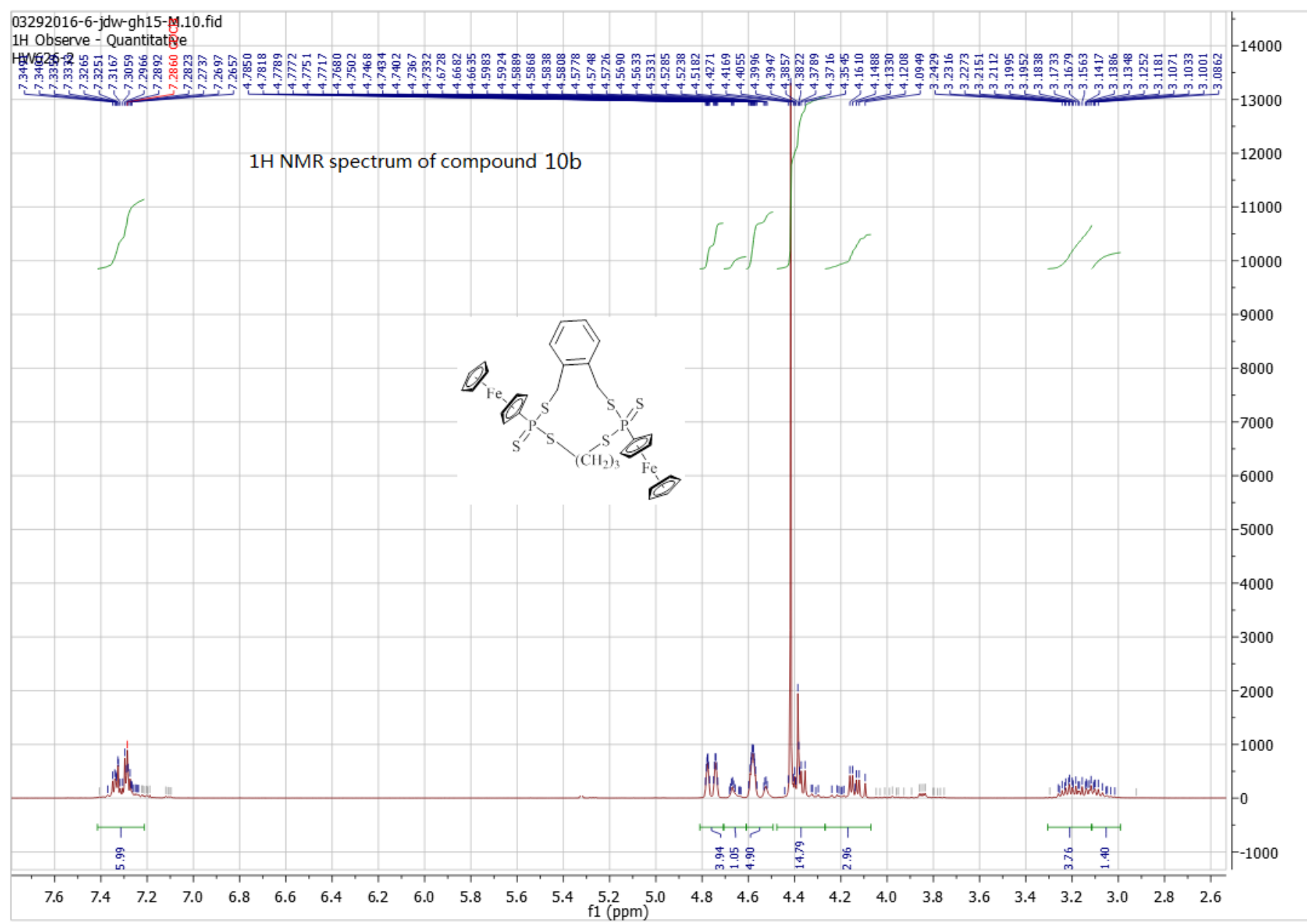




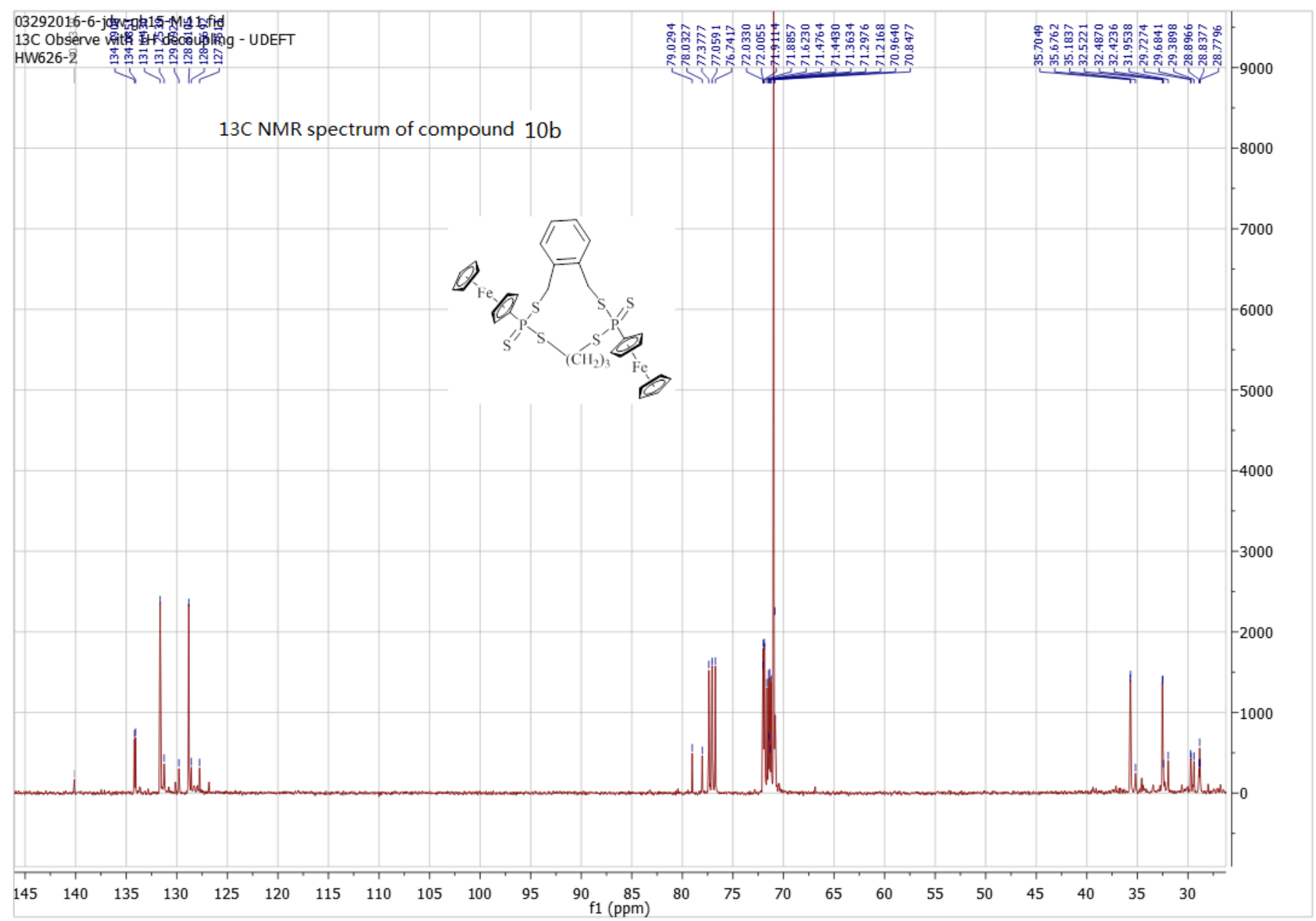




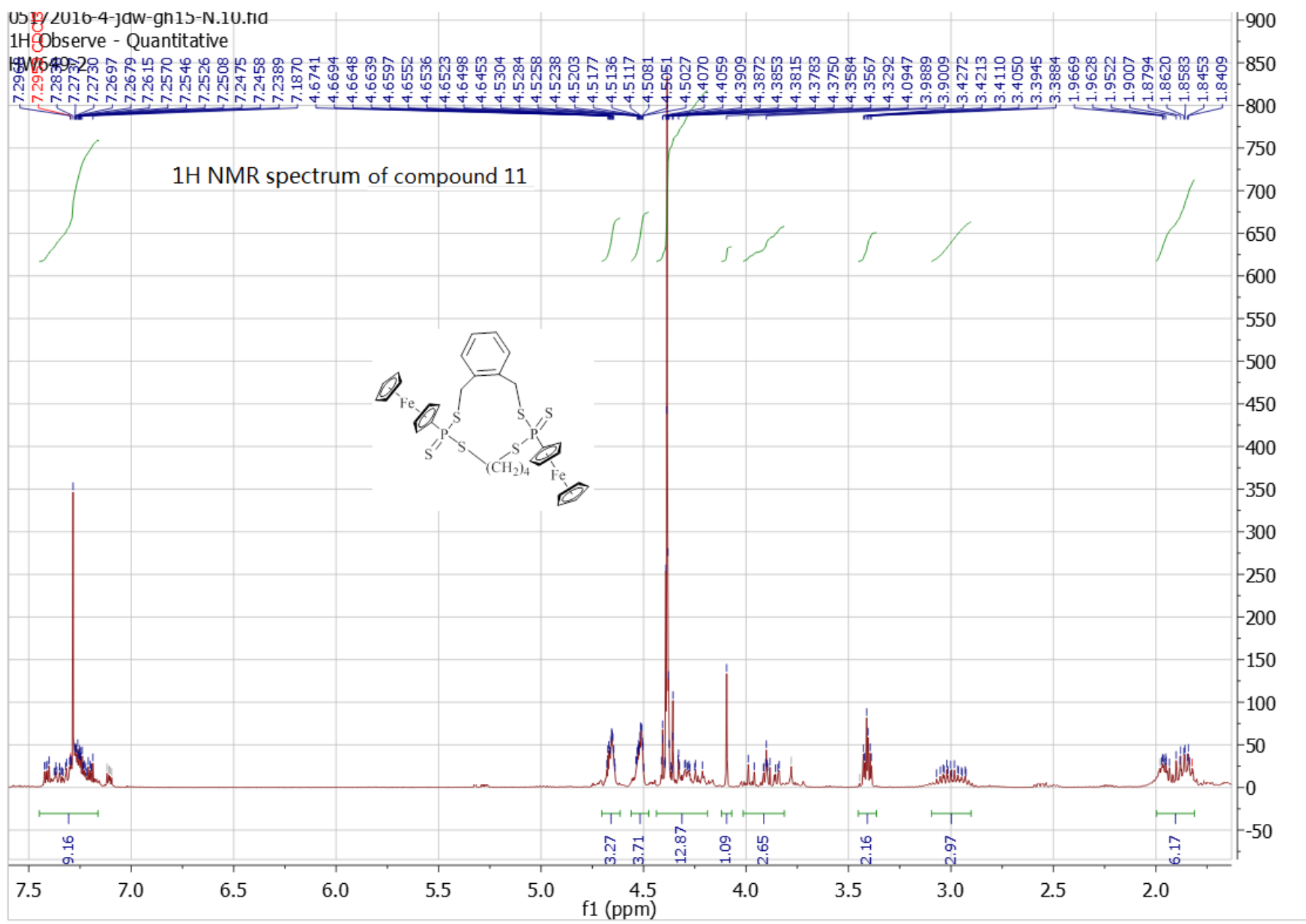




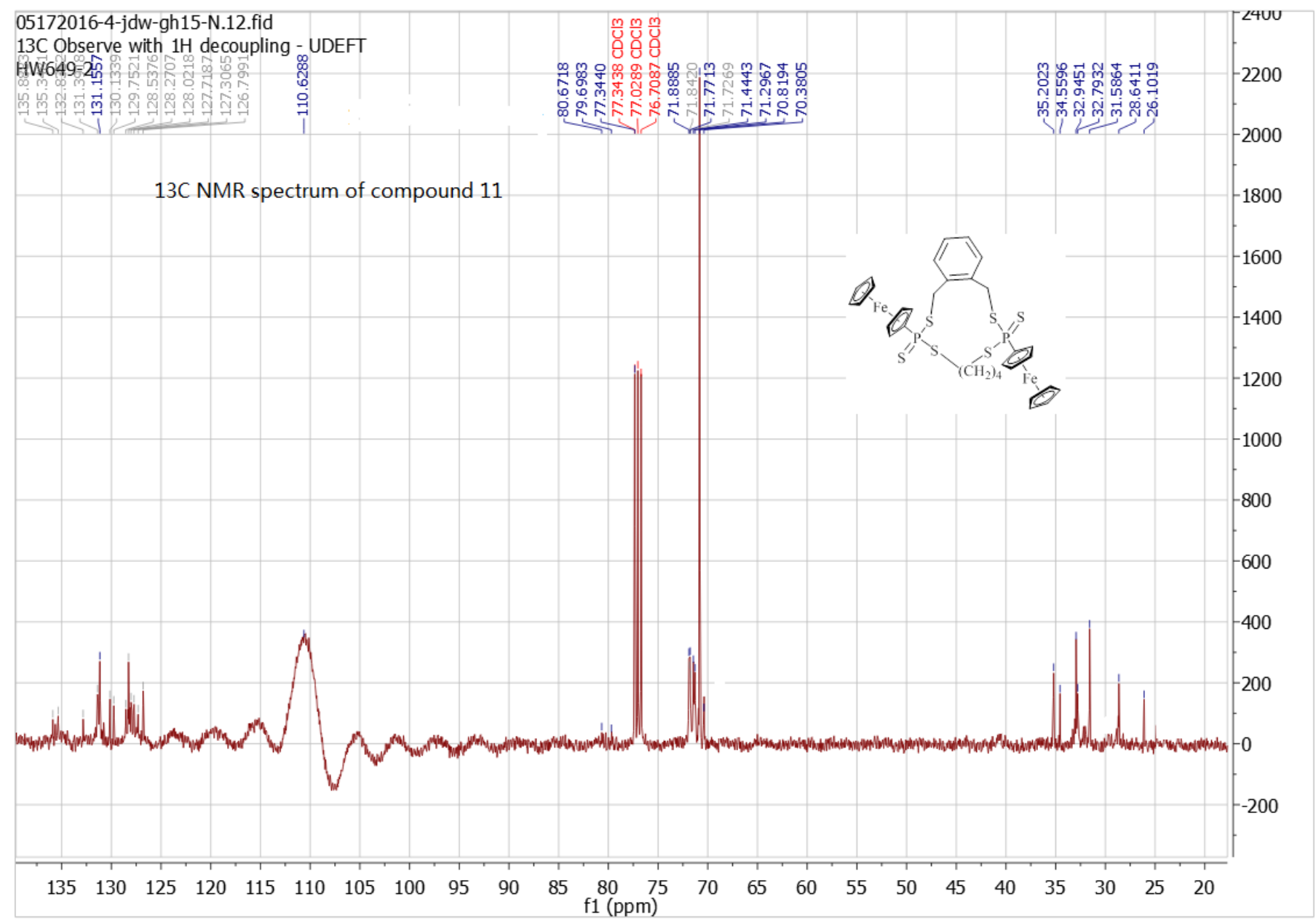




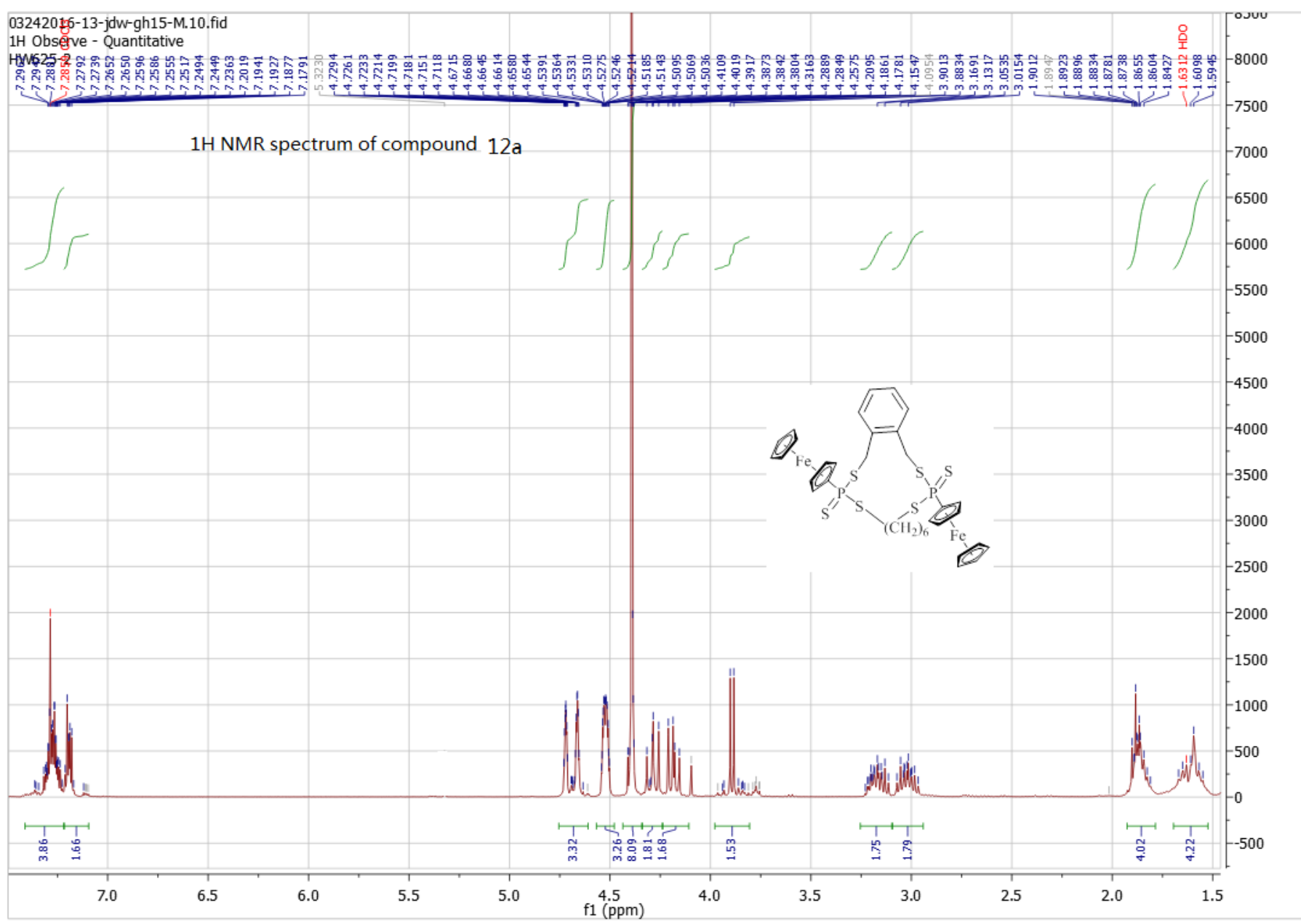




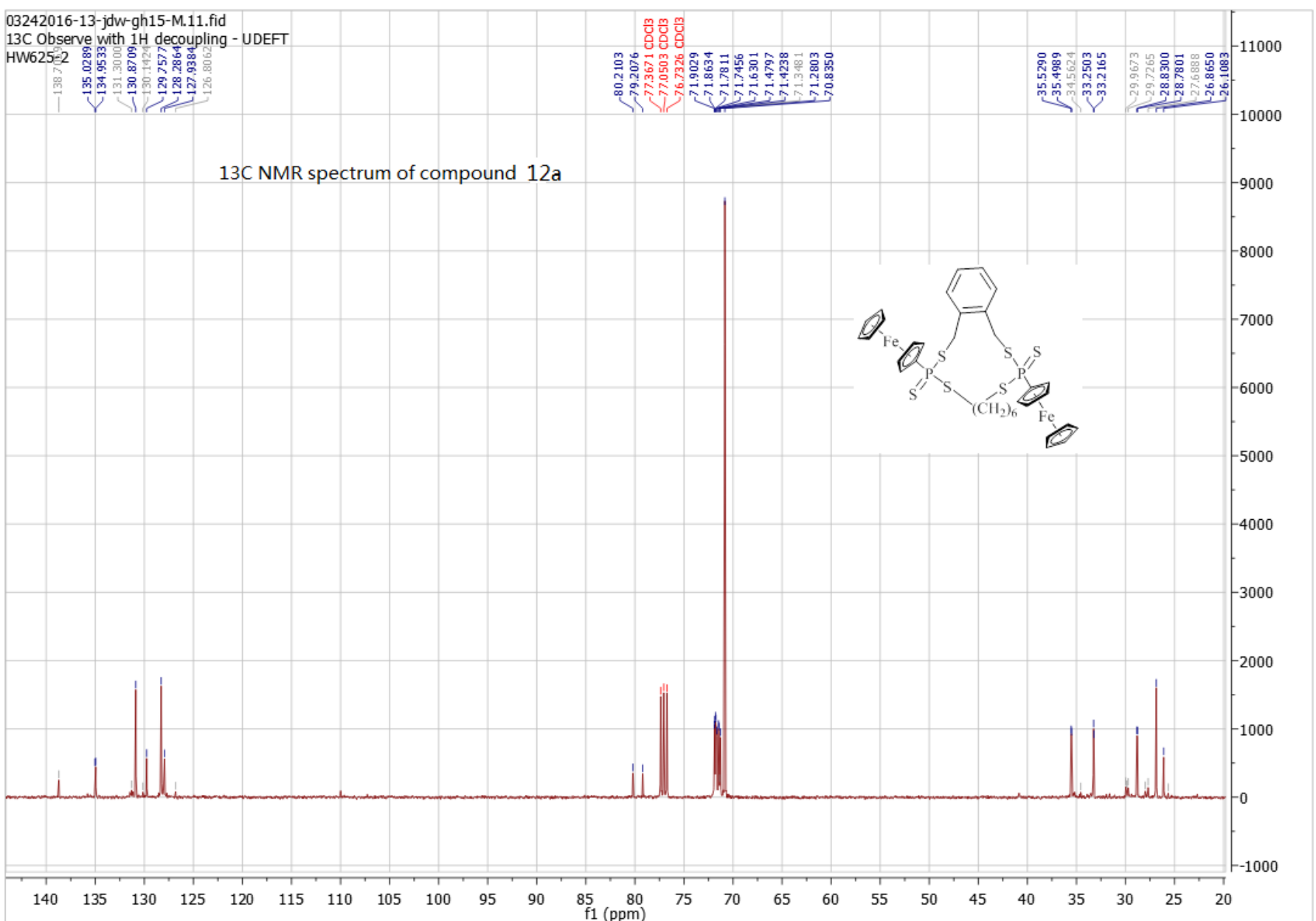




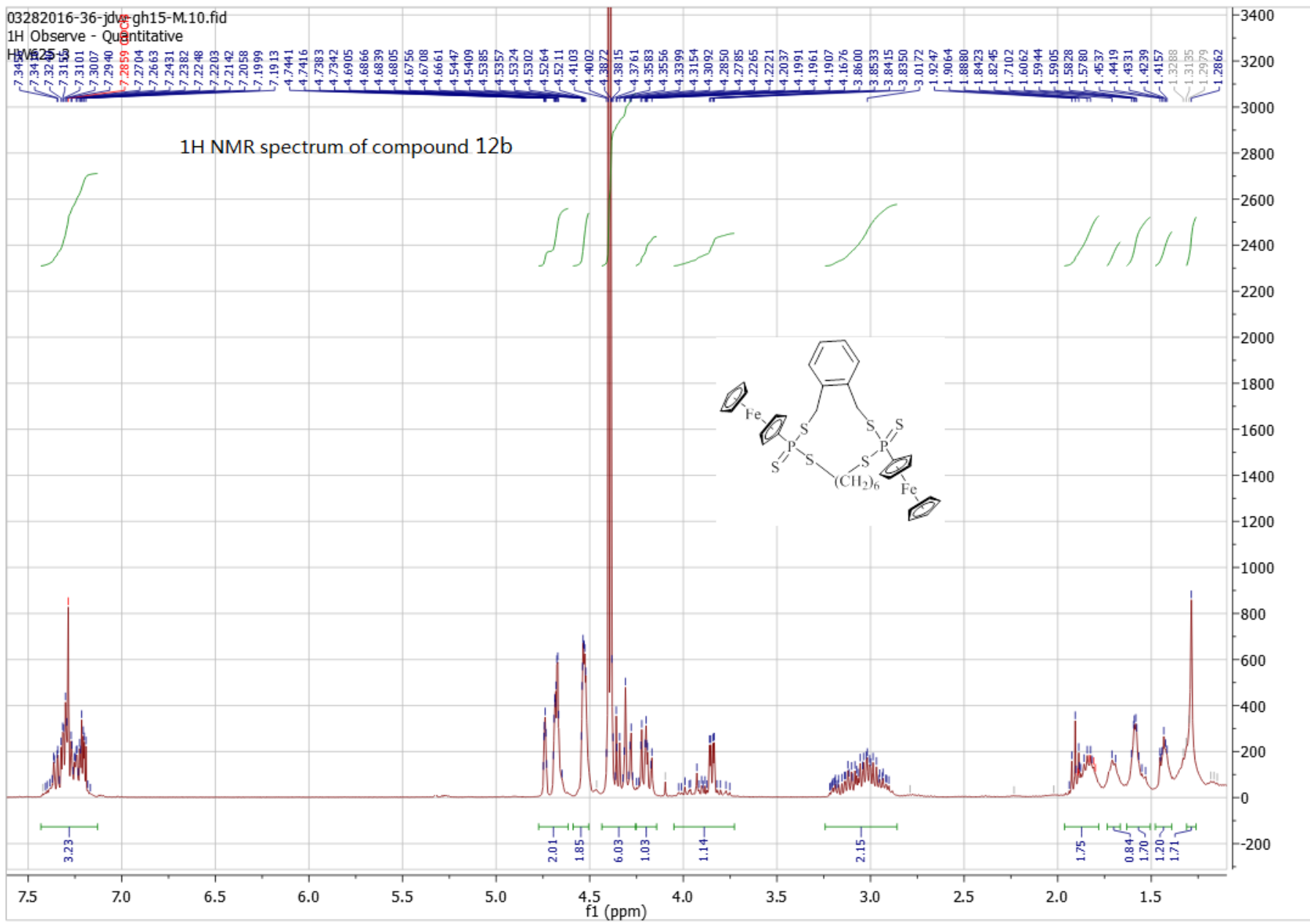




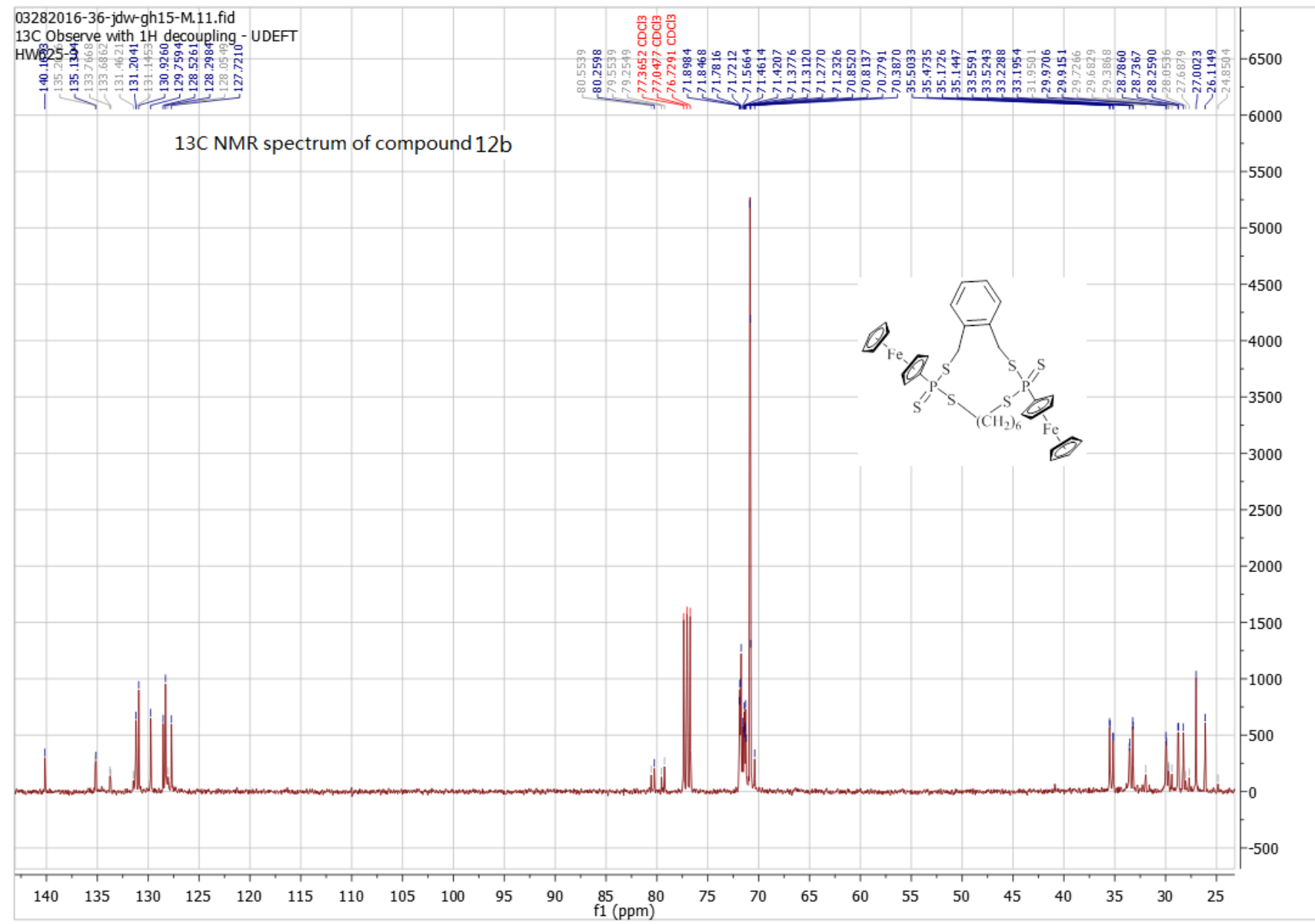




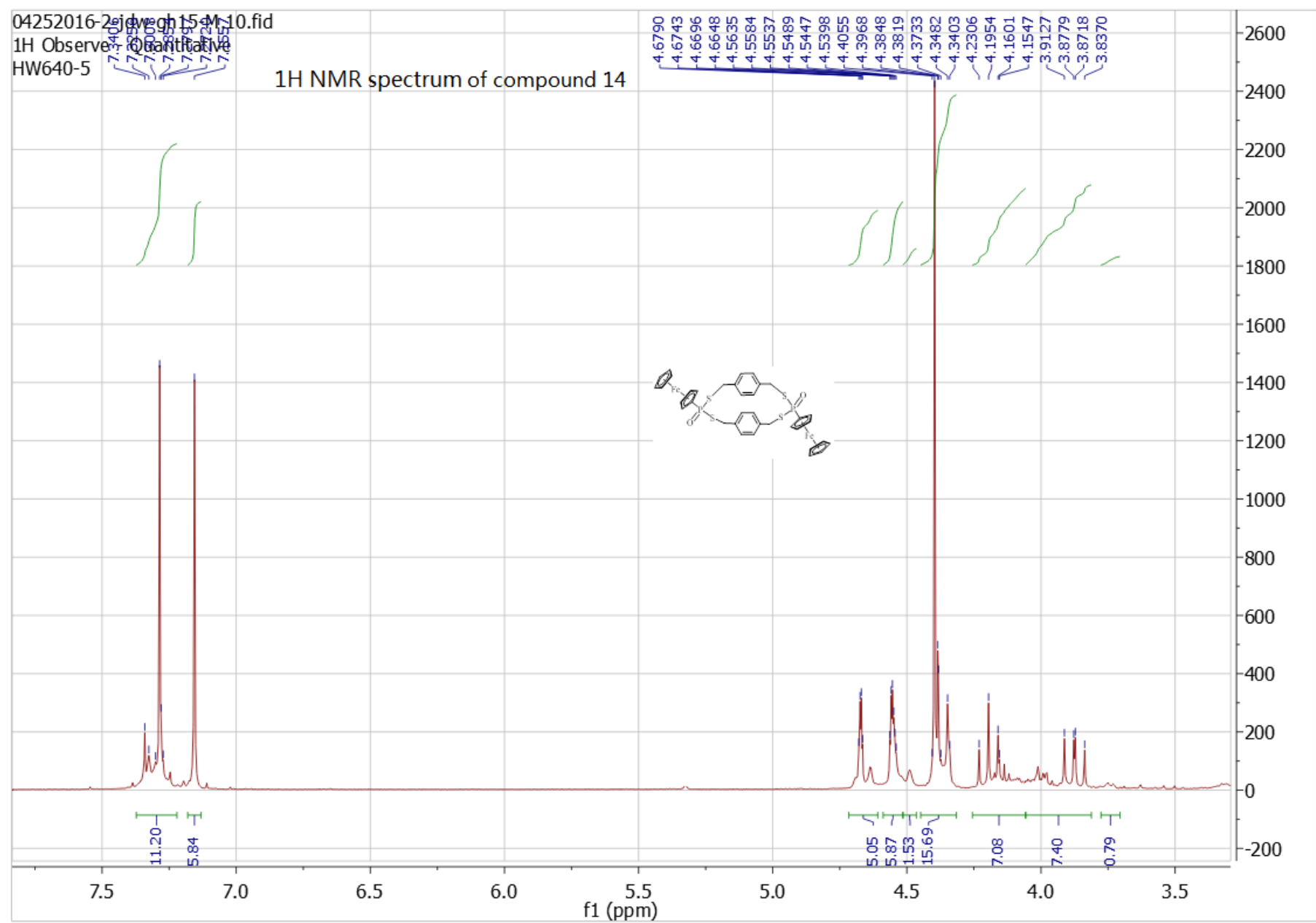




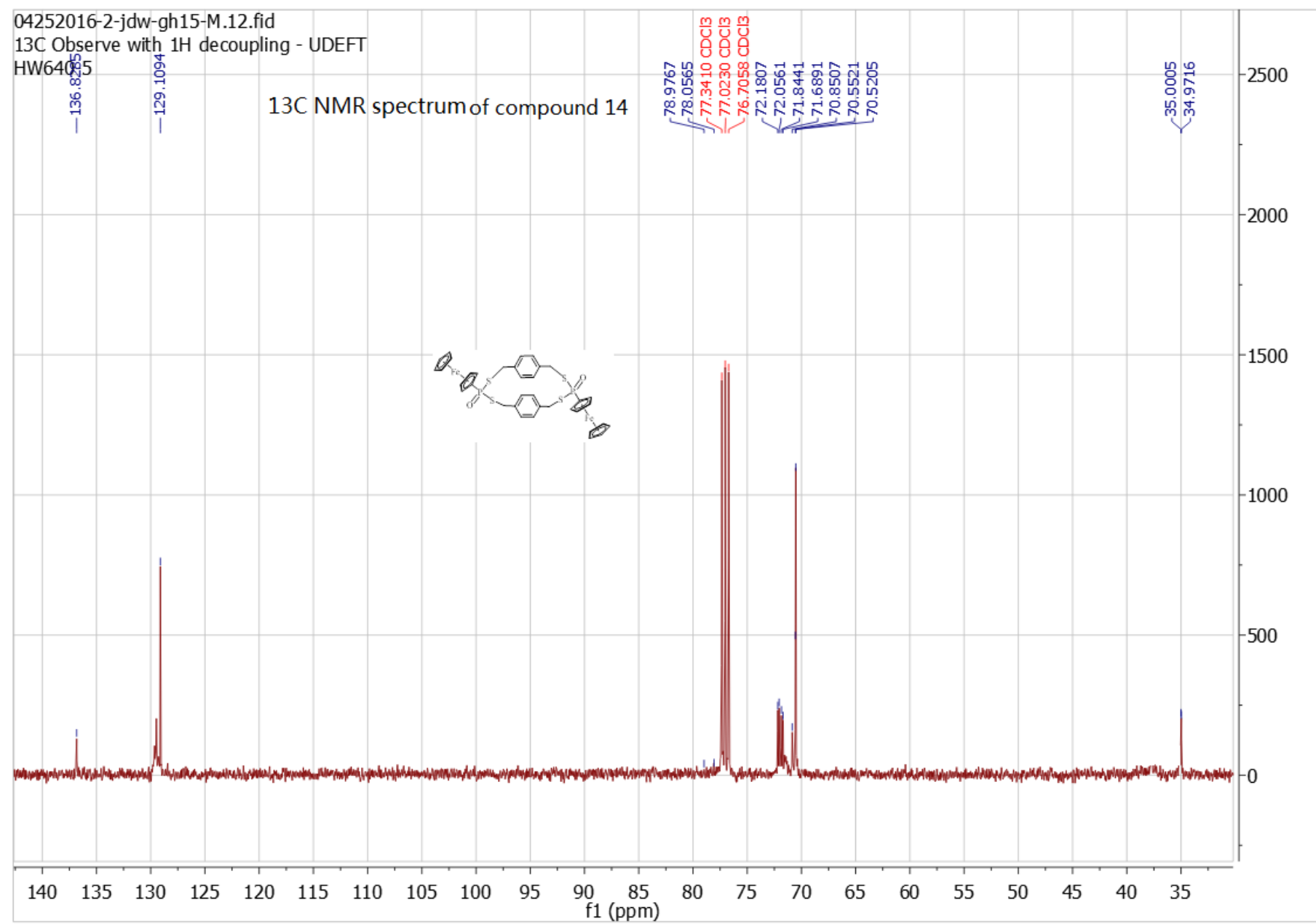

\section{References}

(s1) Foreman, M. R. St. J.; Slawin, A. M. Z.; Woollins, J. D. J. Chem. Soc. Dalton Trans. 1996, 3653.

(s2) Parveen, S.; Kilian, P.; Slawin, A. M. Z.; Woollins, J. D. Dalton Trans. 2006, 2586.

(s3) Fuller, A. L.; Scott-Hayward, L. A. S.; Li, Y.; Bühl, M.; Slawin, A. M. Z.; Woollins, J. D. J. Am. Chem. Soc. 2010, 132, 5799.

(s4) CrystalClear-SM Expert v3.1b27. Rigaku Americas, The Woodlands, Texas, USA, and Rigaku Corporation, Tokyo, Japan, 2013.

(s5) CrystalClear-SM Expert v2.1. Rigaku Americas, The Woodlands, Texas, USA, and Rigaku Corporation, Tokyo, Japan, 2015.

(s6) Palatinus, L.; Chapuis, G. J. Appl. Cryst. 2007, 40, 786.

(s7) Burla, M. C.; Caliandro, R.; Camalli, M.; Carrozzini, B.; Cascarano, G. L.; De Caro, L.; Giacovazzo, C.; Polidori, G.; Spagna, R. J. Appl. Cryst. 2005, 38, 381.

(s8) Burla, M. C.; Caliandro, R.; Camalli, M.; Carrozzini, B.; Cascarano, G. L.; Giacovazzo, C.; Mallamo, M.; Mazzone, A.; Polidori, G.; Spagna, R. J. Appl. Cryst. 2012, 45, 357. 
(s9) Sheldrick, G. M. Acta. Crystallogr., Sect. C. 2015, 71, 3.

(s10) CrystalStructure v4.2. Rigaku Americas, The Woodlands, Texas, USA, and Rigaku Corporation, Tokyo, Japan, 2015. 